\title{
Conformal group theory of tensor structures
}

\author{
Ilija Burić, ${ }^{a}$ Volker Schomerus ${ }^{a}$ and Mikhail Isachenkov ${ }^{b}$ \\ ${ }^{a}$ DESY, Notkestraße 85, Hamburg D-22607, Germany \\ ${ }^{b}$ IHÉS, 35 Route de Chartres, Bures-sur-Yvette 91440, France \\ E-mail: ilija.buric@desy.de, volker.schomerus@desy.de, \\ isachenkov@ihes.fr
}

ABSTRACT: The decomposition of correlation functions into conformal blocks is an indispensable tool in conformal field theory. For spinning correlators, non-trivial tensor structures are needed to mediate between the conformal blocks, which are functions of cross ratios only, and the correlation functions that depend on insertion points in the $d$ dimensional Euclidean space. Here we develop an entirely group theoretic approach to tensor structures, based on the Cartan decomposition of the conformal group. It provides us with a new universal formula for tensor structures and thereby a systematic derivation of crossing equations. Our approach applies to a 'gauge' in which the conformal blocks are wave functions of Calogero-Sutherland models rather than solutions of the more standard Casimir equations. Through this ab initio construction of tensor structures we complete the Calogero-Sutherland approach to conformal correlators, at least for four-point functions of local operators in non-supersymmetric models. An extension to defects and superconformal symmetry is possible.

Keywords: Conformal Field Theory, Global Symmetries, Space-Time Symmetries

ARXIV EPRINT: 1910.08099 


\section{Contents}

1 Introduction 1

2 Correlators as functions on the conformal group 4

2.1 Background, conformal and Weyl inversions 5

$\begin{array}{lll}2.2 & \text { Correlators from covariant functions on the group } & 7\end{array}$

3 Cartan coordinates and tensor structures $\quad 9$

$\begin{array}{lll}3.1 & \text { Cartan coordinates and Calogero-Sutherland models } & 10\end{array}$

$\begin{array}{ll}3.2 & \text { Cartan decomposition and tensor structures } \\ \end{array}$

4 Computation of tensor structures in $d=3,4 \quad 14$

$\begin{array}{ll}4.1 \text { Spinor representation of the conformal group } & 14\end{array}$

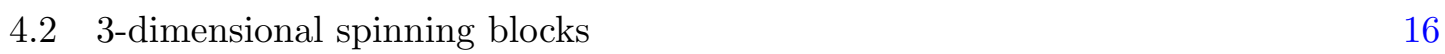

$\begin{array}{lll}\text { 4.2.1 Seed conformal blocks } & 17\end{array}$

$\begin{array}{lll}\text { 4.2.2 Tensor structures for arbitrary spins } & 19\end{array}$

$\begin{array}{ll}4.3 & \text { 4-dimensional spinning seed blocks } 20\end{array}$

5 Comparison with other approaches $\quad 22$

5.13 -dimensional spinning blocks 23

5.2 4-dimensional spinning blocks 25

6 Conclusions and outlook $\quad 27$

A Verification of Ward identities $\quad 28$

$\begin{array}{ll}\text { B Correlators from harmonic analysis } & 31\end{array}$

C Derivation of eqs. (3.9) and (3.12) 33

$\begin{array}{ll}\text { D Euler angles and quaternions } & 34\end{array}$

\section{Introduction}

Conformal partial wave expansions are an indispensable tool in conformal field theory that allows to decompose correlation functions of local and non-local operators into a basis of kinematically determined building blocks. Such decompositions were introduced in the early days of conformal field theory, but the mathematical nature of the blocks remained elusive for a long time. In fact, before the work of Dolan and Osborn [1, 2], the shadow formalism of Ferrara et al. [3] only provided certain integral expressions even for correlation functions of scalar fields. Dolan and Osborn characterized the scalar blocks through second order Casimir differential equations and thereby managed to uncover many of their properties, including explicit formulas for scalar four-point blocks in even dimensions. Boosted by the success of the numerical bootstrap [4-8] the construction of conformal partial wave expansions was extended in many different directions. Analyzing correlators of fields with 
spin, such as e.g. currents or the stress tensor, is clearly one of the most pressing issues that was addressed early on in [9-13], see also $[14,15]$ and references therein for more recent developments.

While correlation functions depend on insertion points $x_{i}$, conformal blocks are functions of cross ratios $z_{i}$ only. Hence, any conformal partial wave expansion requires inserting a properly chosen factor that can turn functions of conformally invariant cross ratios into functions of insertion points in such a way that conformal Ward identities are satisfied. This factor $\Theta$, usually referred to as "tensor structures", may be considered as a rectangular matrix whose entries are functions of the insertion points. One index of the matrix $\Theta$ collects all the tensor indices of the involved fields while the other runs through some basis in the space of tensor structures. Conformal Ward identities do not determine the tensor structures uniquely since they are left invariant if we multiply these by a matrix of functions of the cross ratios. This inherent gauge freedom is usually fixed by making some more or less convenient or simple choices before working out the Casimir differential equations and constructing the associated conformal blocks, see e.g. [16, 17] for some examples.

In [18] the authors noticed that the Casimir equations for scalar four-point functions derived by Dolan and Osborn could be (gauge) transformed into stationary Schroedinger equations for integrable two-particle Hamiltonians of Calogero-Sutherland type. The latter have been studied extensively in the mathematical literature, starting with the work of Heckman and Opdam [19]. The connection uncovered in [18] implies that conformal blocks are certain multivariable hypergeometric functions with properties that follow from integrability of the quantum mechanical system and are very similar to those of ordinary hypergeometrics in one variable. Later, it was understood that the relation between conformal blocks and Calogero-Sutherland models is entirely universal. More concretely, for all spinning correlators there exists some gauge in which the associated Casimir equations possess Calogero-Sutherland form, see [20, 21]. In contrast to the standard approach we described above, the associated Casimir equations could be constructed without ever fixing particular tensor structures $\Theta$. This underlines the canonical nature of the Calogero-Sutherland gauge in comparison to all other choices and it has made it easy to detect relations between seemingly different setups, e.g. between Casimir equations for four-point functions and defect correlators [22], and to apply standard solution generating techniques [23, 24] to study new types of blocks.

In all cases in which Casimir equations for spinning conformal blocks had been known, they were shown by explicit computations to be equivalent to eigenvalue equations for spinning Calogero-Sutherland Hamiltonians. The equivalence is mediated by a specific gauge transformation that relates the two sets of Casimir equations. Once the gauge transformation is known it can be used to establish a direct relation between blocks in Calogero-Sutherland gauge and correlation functions since tensor structures for many kinds of spinning blocks had been worked out explicitly. On the other hand, it is certainly not satisfactory that so far the explicit relation between correlation functions and CalogeroSutherland wave functions was composed from previously constructed tensor structures and a gauge transformation between usual Casimir equations and Calogero-Sutherland eigenvalue equations. More importantly, in the context of superblocks, there exists a 
large class of cases now in which the Calogero-Sutherland eigenvalue equations are known while tensor structures and the associated Casimir equations have not been constructed. In those cases a direct construction of tensor structures in Calogero-Sutherland gauge is indispensable. Let us note that also the conformal bootstrap programme cannot do without this. Even though the crossing symmetry constraints are usually written for functions of cross ratios only, knowledge of $s$ - and $t$-channel blocks is not sufficient. To set up the relevant equations one also has to compute the ratio of $s$ - and $t$-channel tensor structures. The resulting crossing factor is a non-trivial function of the cross ratios that must be taken into account when solving for the dynamical content of the theory.

All this provides strong motivation for the present work in which we will construct a direct map between correlation functions and Calogero-Sutherland wave functions. We have explained above that Calogero-Sutherland eigenvalue equations arise when we write Casimir equations for conformal blocks in a distinguished gauge, one that is deeply routed in the group theory of the conformal group. In this context, we may rephrase the main result of this work as a direct construction of tensor structures in the very same canonical Calogero-Sutherland gauge. As we shall see below, tensor structures in the CalogeroSutherland gauge arise from a change of coordinates on the conformal group. This group theoretic understanding will allow us to provide universal formulas for tensor structures. More concretely we will prove that any spinning four-point correlation function $G$ possesses a canonical factorization of the form

$$
G\left(\pi_{i} ; x_{i}\right)=\tilde{\Omega}\left(\Delta_{i} ; x_{i}\right) \Theta\left(\lambda_{i} ; x_{i}\right) \psi\left(\pi_{i} ; u_{1}, u_{2}\right) .
$$

Here $\pi_{i}=\left(\Delta_{i}, \lambda_{i}\right)$ denote the conformal weights $\Delta_{i}$ and spins $\lambda_{i}$ of the four fields. The prefactor $\tilde{\Omega} \Theta$ is split off from the correlation function $G$ so that $\psi$ depends on the insertion points $x_{i}$ only through the usual cross ratios $z=z_{1}$ and $\bar{z}=z_{2}$, see eq. (3.10). The arguments $u_{i}$ we display here are related to these cross ratios as $z_{i}=-\sinh ^{-2} u_{i} / 2$. Our concrete choice for $\tilde{\Omega} \Theta$, see next paragraph, ensures that $\psi$ can be expanded in eigenfunctions of some matrix valued Calogero-Sutherland Hamiltonian with constant coefficients. The eigenvalue equation for the Calogero-Sutherland Hamiltonian takes the place of the conventional Casimir equations for conformal blocks $g$.

The prefactor $\tilde{\Omega} \Theta$ is composed from two factors, one scalar factor $\tilde{\Omega}$ that depends only on the conformal weights $\Delta_{i}$ and another matrix valued factor that is sensitive to the spins $\lambda_{i}$ and becomes trivial in case all four fields are scalar. Put differently, while $\tilde{\Omega}$ is associated with the transformation under elements of the dilation group $D=\mathrm{SO}(1,1)$, the tensor structures $\Theta$ mediate the transformation behavior with respect to the action of the (double covering of the) rotation group $R=\operatorname{Spin}(d)$. Our choice of the function $\tilde{\Omega}$ is easy to spell out explicitly. In the $s$-channel, for example, it takes the form

$$
\tilde{\Omega}_{s}\left(x_{i}\right)=\frac{\frac{1}{2}(-1)^{\frac{d-2}{2}}}{x_{12}^{\Delta_{1}+\Delta_{2}} x_{34}^{\Delta_{3}+\Delta_{4}}}\left(\frac{x_{14}}{x_{24}}\right)^{2 a}\left(\frac{x_{14}}{x_{13}}\right)^{2 b} \frac{\left(z_{1} z_{2}\right)^{\frac{d-1}{2}}\left|z_{1}-z_{2}\right|^{-\frac{d-2}{2}}}{\left[\left(1-z_{1}\right)\left(1-z_{2}\right)\right]^{\frac{a}{2}+\frac{b}{2}+\frac{1}{4}}},
$$

where $2 a=\Delta_{2}-\Delta_{1}$ and $2 b=\Delta_{3}-\Delta_{4}$. This $\tilde{\Omega}$ differs from the function $\Omega$ that was introduced in [2] by some specific function of the cross ratios which was first constructed 
in [18]. The most novel part of our formula (1.1) is the construction of the factor $\Theta$. In order to obtain the matrix of tensor structures $\Theta=\Theta_{s}$ in the $s$-channel, for example, we consider the following family of elements of the conformal group,

$$
g\left(x_{i}\right)=e^{\frac{x_{21}^{\mu}}{x_{21}^{2}} K_{\mu}} e^{x_{13}^{\mu} P_{\mu}} e^{\frac{x_{34}^{\mu}}{x_{34}^{2}} K_{\mu}}=d_{l}\left(x_{i}\right) r_{l}\left(x_{i}\right) e^{\frac{u_{1}+u_{2}}{4}\left(P_{1}+K_{1}\right)-i \frac{u_{1}-u_{2}}{4}\left(P_{2}-K_{2}\right)} d_{r}\left(x_{i}\right) r_{r}\left(x_{i}\right) .
$$

It turns out that all elements of the conformal group, up to a set of Haar measure zero, can be written as a product of a dilation $d_{l} \in \mathrm{SO}(1,1)$, a rotation $r_{l} \in \operatorname{Spin}(d)$, an element $a$ of a particular 2-dimensional abelian subgroup and then another dilation $d_{r}$ and rotation $r_{r}$, see the right hand side of the previous equation. This decomposition defines two functions $r_{l}\left(x_{i}\right)$ and $r_{r}\left(x_{i}\right)$ of the insertion points that take values in the subgroup $R$ of rotations. When these are represented in some finite dimensional representations of the rotation group, one that is determined by the set of spins $\lambda_{i}$, we obtain the tensor structures $\Theta_{s}$. A concrete formula is stated in eq. (3.16) with a prefactor $\hat{\rho}$ defined in eq. (2.16).

Let us now describe the plan of this paper in more detail. In the next section we shall explain how to map conformal four-point correlation functions to a special class of covariant functions on the conformal group, which may be appropriately called $K$-spherical functions. This map is given in (2.14). Once the correlator is lifted to the group, we can work out the Casimir equations through the restriction of the Laplace operator to this class of functions. In section 3, we introduce a particular set of coordinates on the group which are very well suited for the study of $K$-spherical functions. These coordinates, which may be considered as higher dimensional generalizations of the usual Euler angles for $\mathrm{SO}(3)$, arise from a KAK, or Cartan, decomposition of the conformal group. Thus we shall often call them Cartan coordinates. The Laplacian assumes a particularly simple form in Cartan coordinates, when acting on $K$-spherical functions, and reduces to the CalogeroSutherland Hamiltonian mentioned before. In this context, the factors appearing in (1.1) simply arise from a change of coordinates on the conformal group. Namely, in section 2 we parametrize a family of group elements through the insertion points of our local fields, as stated in eq. (1.3). The transformation to Cartan coordinates is a group theoretic problem that, once it is solved, provides universal formulas for the tensor structures, for any given assignment of spins, written in (3.16). After the general theory is explained in section 3, we describe the explicit solution and computation of tensor structures for the conformal groups in $d=3$ and $d=4$ dimensions in section 4 . In section 5 we evaluate the tensor structures for some special choices of spins to compare with previous expressions in the literature, most notably in [16] and [17], finding agreement in all cases. Our work concludes with an outlook. In particular we compute the crossing factor, which appears in the crossing symmetry equations, for the simplest spinning seed blocks in $d=4$ dimensions and observe that is takes a very simple form. In addition we comment briefly on possible extensions to superblocks and defects.

\section{Correlators as functions on the conformal group}

In this section, our main goal is to reinterpret four-point correlation functions of local primary fields in a conformal field theory in terms of functions on the conformal group which 
obey certain covariance properties. In the first subsection we collect some background material on primary fields, correlation functions and Ward identities. The discussion includes a review of conformal and Weyl inversions. The main result of the section is stated at the beginning of the second subsection, (2.14), along with a sketch of the derivation and some additional comments on the relation with embedding space constructions. A full proof of our result can be found in appendices A and B. Applications of the formula (2.14) are left for the next section.

\subsection{Background, conformal and Weyl inversions}

A local primary field $\Phi_{(\Delta, \lambda)}$ in conformal field theory is characterized by its weight $\Delta$ and its spin $\lambda$. In representation theoretic terms, the weight and spin correspond to a finite dimensional representation $\pi=(\Delta, \lambda)$ of the product group $K=\operatorname{SO}(1,1) \times \operatorname{Spin}(d) \subset G=$ $\operatorname{Spin}(1, d+1)$. By definition, the transformation behavior of such a conformal primary field with respect to infinitesimal conformal transformations is given by

$$
\begin{aligned}
{\left[D, \Phi_{\pi}(x)\right] } & =\left(x^{\nu} \partial_{\nu}+\Delta\right) \Phi_{\pi}(x) \\
{\left[L_{\mu \nu}, \Phi_{\pi}(x)\right] } & =\left(x_{\nu} \partial_{\mu}-x_{\mu} \partial_{\nu}+\Sigma_{\mu \nu}^{\lambda}\right) \Phi_{\pi}(x) \\
{\left[K_{\mu}, \Phi_{\pi}(x)\right] } & =\left(x^{2} \partial_{\mu}-2 x_{\mu} x^{\nu} \partial_{\nu}-2 x_{\mu} \Delta+2 x^{\nu} \Sigma_{\mu \nu}^{\lambda}\right) \Phi(x)
\end{aligned}
$$

where $\Sigma_{\mu \nu}^{\lambda}=\pi\left(L_{\mu \nu}\right)$ denote the representation matrices of the generators $L_{\mu \nu}$ of rotations in the representation $\lambda$ of $\operatorname{Spin}(d)$. The generators of translations act simply as derivatives.

It is important for us to understand the action of the conformal inversion in some detail. The conformal inversion $I: \mathbb{S}^{d} \rightarrow \mathbb{S}^{d}$ acts on the coordinates of the conformally compactified Euclidean space $\mathbb{S}^{d}$ as

$$
I\left(x^{\mu}\right)=\frac{x^{\mu}}{x^{2}}
$$

In order to determine the transformation law of primary fields under the conformal inversion, we are instructed to compute the differential (Jacobian) of $I$, which takes the form

$$
d I_{\nu}^{\mu}(x)=\partial_{\nu} \frac{x^{\mu}}{x^{2}}=\frac{1}{x^{2}}\left(\delta_{\nu}^{\mu}-2 \hat{x}^{\mu} \hat{x}_{\nu}\right)=\frac{1}{x^{2}} s_{x} .
$$

Thus the Jacobian $d I$ decomposes as the product of a conformal factor $x^{-2}$ and a reflection $s_{x}$ in the hyperplane orthogonal to $x$. The inversion $I$ can be thought of as an element of $\operatorname{Pin}(1, d+1)$ with $\operatorname{det}(I)=-1$, i.e. it does not belong to the identity component of $\operatorname{Pin}(1, d+1)$. Explicitly, the inversion $I$ takes the form $I=\operatorname{diag}(1,-1,1, \ldots, 1)$. In a parityinvariant conformal field theory, a primary field which transforms in the representation $\pi$ of the orthogonal group $O(d)$ is acted on by $I$ as

$$
\Phi^{I}(x)=I \Phi(x) I^{-1}=\left(\frac{1}{x^{2}}\right)^{-\Delta} \pi\left(s_{x}\right) \Phi(I(x)) .
$$

Since in a parity-invariant theory fields only transform in those representations $\pi$ of the rotation group $\operatorname{Spin}(d)$ which extend to $\operatorname{Pin}(d)$, the representation matrix $\pi\left(s_{x}\right)$ is welldefined. To describe the transformation behavior in a general conformal field theory, we 
introduce the reflections $s_{e_{i}}$ along the hyperplane orthogonal to $e_{i}$

$$
s_{e_{i}}:\left(x_{1}, \ldots, x_{i}, \ldots, x_{n}\right) \mapsto\left(x_{1}, \ldots,-x_{i}, \ldots, x_{n}\right)
$$

With the help of these reflections, we can now define new maps $I_{i}=I s_{e_{i}}=s_{e_{i}} I$ which are in the identity component $\operatorname{Spin}(1, d+1)$ of the conformal group. Their differentials are given by

$$
d I_{i}(x)=\frac{1}{x^{2}} s_{e_{i}} s_{x}
$$

We will denote the group element $I_{d}$ by $w$ and call it the Weyl inversion. Under the action of $w$, a primary field transforms as

$$
\Phi^{w}(x)=\left(\frac{1}{x^{2}}\right)^{-\Delta} \pi\left(s_{e_{d}} s_{x}\right) \Phi(w(x)) .
$$

We note that the product $s_{e_{d}} s_{x}$ of two reflections is a proper rotation and hence its representation matrix $\pi\left(s_{x} s_{e_{d}}\right)$ is well defined for all representations $\pi$ of the spin group. For later use let us note that the Weyl inversion $w$ can be used to construct a new representation $\pi^{\prime}=\pi^{w}$ of the subgroup $K$ from $\pi$ through

$$
\pi^{\prime}(k):=\pi\left(w k w^{-1}\right)
$$

for all $k \in K$. By construction $w k w^{-1} \in K$ and hence these elements can be evaluated in $\pi$ so that the right hand side is well defined. In general, the representations $\pi$ and $\pi^{\prime}$ are inequivalent. We also observe that

$$
\pi^{\prime}(D)=-\pi(D)
$$

This concludes our short detour on conformal inversions and their action on primary fields in a general conformal field theory.

Let us now choose four representations $\pi_{i}=\left(\Delta_{i}, \lambda_{i}\right), i=1, \ldots, 4$, one for each primary field we want to insert, and consider the correlation function

$$
G\left(\pi_{i}, x_{i}\right)=\left\langle\Phi_{\pi_{1}}\left(x_{1}\right) \ldots \Phi_{\pi_{4}}\left(x_{4}\right)\right\rangle
$$

We shall think of this as a function from the set of insertion points $x_{i}$ to the space

$$
W_{P}=\bigotimes_{i=1}^{4} V_{i}
$$

of polarisations. Here, $V_{i}$ denotes the finite dimensional carrier space of the representation $\pi_{i}$ of $K$. Conformal invariance implies that the four-point functions $G$ satisfy a set of differential Ward identities which take the form

$$
\left\langle\left[X, \Phi_{\pi_{1}}\left(x_{1}\right) \ldots \Phi_{\pi_{4}}\left(x_{4}\right)\right]\right\rangle=0
$$


for all generators $X$ of the conformal algebra. The commutator of $X$ with the individual primary fields can be evaluated using the familiar commutation relations (2.2). In the case of dilations, i.e. for $X=D$, for example, the Ward identity reads

$$
\sum_{i=1}^{4} x_{i}^{\nu} \partial_{\nu}^{(i)} G\left(x_{i}\right)=-\sum_{i=1}^{4} \Delta_{i} G\left(x_{i}\right)
$$

where $\partial^{(i)}$ denotes the derivative with respect to the insertion point $x_{i}$. For the other generators, the Ward identities take a similar form.

\subsection{Correlators from covariant functions on the group}

Our goal in this subsection is to show that, for any given choice of a channel, the solutions of the Ward identities are in 1-1 correspondence with certain covariant functions on the conformal group. We will first discuss this for the $s$-channel and comment on other channels at the end.

The representation theoretic data we have introduced, i.e. the four representations $\pi_{i}=\left(\Delta_{i}, \lambda_{i}\right)$ of the subgroup $K$, determine a subspace $\Gamma^{\pi_{i}}$ of functions on the conformal group that take values in the space $W_{P}$ of polarisations. In order to characterize the relevant space of functions we introduce the representation $\hat{\pi}_{\sigma}$ as ${ }^{1}$

$$
\begin{aligned}
& \hat{\pi}_{s}\left(k_{l}, k_{r}\right)=\pi_{12}\left(k_{l}\right) \otimes \pi_{34}\left(k_{r}^{-1}\right) \quad \text { for }\left(k_{l}, k_{r}\right) \in K \times K \\
& \text { where } \pi_{i j}(k)=\pi_{i}(k) \otimes \pi_{j}^{\prime}(k) \quad \text { for } \quad k \in K \text {. }
\end{aligned}
$$

Here the subscript $s$ simply reminds us that we are in the $s$ channel. With this notation we can now define the linear subspace $\Gamma^{\pi_{i}}$ to consist of all those $W_{P}$-valued functions on the conformal group which satisfy the covariance laws

$$
F_{s}\left(k_{l} g k_{r}\right)=\hat{\pi}_{s}\left(k_{l}, k_{r}\right) F_{s}(g)
$$

Within this subspace we can now find a unique representative of the correlation function $G\left(x_{i}\right)$. More precisely, given any solution $G\left(x_{i}\right)$ of the Ward identities, there exists a unique function $F_{s} \in \Gamma^{\pi_{i}}$ such that

$$
G\left(x_{i}\right)=\frac{1}{x_{12}^{2 \Delta_{2}} x_{34}^{2 \Delta_{4}}}\left(1 \otimes \pi_{2}\left(s_{x_{12}} s_{e_{d}}\right) \otimes 1 \otimes \pi_{4}\left(s_{x_{34}} s_{e_{d}}\right)\right) F_{s}\left(e^{I x_{21} \cdot K} e^{x_{13} \cdot P} e^{I x_{34} \cdot K}\right) .
$$

We establish our claim in two steps. First we need to show that the function on the right hand side of eq. (2.14) does indeed satisfy all Ward identities. Once we are done with this step it remains to convince ourselves that all solutions of the Ward identities admit a representation of the form (2.14). Here we want to carry out the first step in the case of translations, dilations and rotations, postponing the full proof to appendix A. Translation symmetry is manifest since the right hand side only depends on differences of insertion

\footnotetext{
${ }^{1}$ Here and in the following the products such as $K \times K$ of subgroups of $G$ are understood as products over $\mathbb{Z}_{2}$, where $\mathbb{Z}_{2}$ is a kernel of the projection $\operatorname{Spin}(1, d+1) \rightarrow \mathrm{SO}_{e}(1, d+1)$.
} 
points. In order to derive the Ward identity (2.10) for dilations one makes use of the fact that

$$
\sum_{i=1}^{4} x_{i}^{\nu} \partial_{\nu}^{(i)} e^{x_{13} \cdot P}=\left[D, e^{x_{13} \cdot P}\right], \quad \sum_{i=1}^{4} x_{i}^{\nu} \partial_{\nu}^{(i)} e^{I x_{21} \cdot K}=\left[D, e^{I x_{21} \cdot K}\right] .
$$

Hence, the action of the derivatives on the argument of the function $F_{s}$ may be written as a commutator with the dilation generator. Both terms of the commutator can be evaluated using an infinitesimal version of the covariance law (2.13) along with the property (2.6) so that we obtain

$$
\sum_{i=1}^{4} x_{i}^{\nu} \partial_{\nu}^{(i)} F_{s}\left(e^{I x_{21} \cdot K} e^{x_{13} \cdot P} e^{I x_{34} \cdot K}\right)=\left(-\Delta_{1}+\Delta_{2}-\Delta_{3}+\Delta_{4}\right) F_{s}\left(e^{I x_{21} \cdot K} e^{x_{13} \cdot P} e^{I x_{34} \cdot K}\right)
$$

where we also inserted $\pi_{i}(D)=-\Delta_{i}$. With the additional contributions from the coefficient of $F_{s}$ in eq. (2.14) we indeed find the desired Ward identity (2.10). The Ward identities for rotations can be verified in the same way. Once again we act with the infinitesimal generators of rotations on the right hand side of equation (2.14). There are three terms that contribute, namely the reflections $s_{x_{12}}$ and $s_{x_{34}}$ in the prefactor and the argument of $F_{s}$. As in our discussion of dilations, the action on the argument of $F_{s}$ can be represented through a commutator with the generator $L_{\mu \nu}$. The latter may be pulled out of the argument using the covariance law (2.13). After this, two of the representation matrices, namely $\Sigma_{\mu \nu}^{2}$ and $\Sigma_{\mu \nu}^{4}$ are conjugated with the element $w$. Since the conformal inversion commutes with rotations, we can replace the conjugation with $w$ by a conjugation with the reflection $s_{e_{d}}$. This conjugation with $s_{e_{d}}$ is removed when we commute these matrices past $\pi_{2}\left(s_{e_{d}}\right)$ and $\pi_{4}\left(s_{e_{d}}\right)$. Commuting the representation matrices past the $x$-dependent reflections yields two terms which cancel those that were obtained by acting the infinitesimal generators on the prefactor. Putting all this together one can show that the right hand side of eq. (2.14) behaves in the same way under rotations as the correlator $G$. More details can be found in appendix A which contains a full proof of the conformal Ward identities for the right hand side of eq. (2.14). The second step in our proof, namely to establish existence of an appropriate function $F_{s}$ for any choice of the correlation function $G$, is carried out in appendix B.

Let us briefly comment on how our formula (2.14) generalizes to other channels. We think of a channel $\sigma$ as a permutation $\sigma \in S_{4}$ of the four fields. The permutation group $S_{4}$ consists of $\left|S_{4}\right|=4 !=24$ elements. In writing our equation (2.14), we have made the choice to pair the as $(1,2)$ and $(3,4)$. The permutations $\sigma=(1,2), \sigma=(3,4)$ and $\sigma=(1,3)(2,4)$ generate a subgroup of order eight that do not affect this choice. The quotient of $S_{4}$ with respect to this subgroup consists of three classes. We shall denote these by $s, t$ and $u$ with $\sigma_{s}=\mathrm{id}, \sigma_{t}=(2,4)$ and $\sigma_{u}=(2,3)$. For non-trivial permutation, our main formula reads

$$
G\left(x_{i}\right)=\frac{1}{x_{\sigma(1) \sigma(2)}^{2 \Delta_{\sigma(2)}} x_{\sigma(3) \sigma(4)}^{2 \Delta_{\sigma(4)}}} \hat{\rho}_{\sigma}\left(x_{i}\right) F_{\sigma}\left(e^{I x_{\sigma(2) \sigma(1)} \cdot K} e^{x_{\sigma(1) \sigma(3)} \cdot P} e^{I x_{\sigma(3) \sigma(4)} \cdot K}\right)
$$

where $\sigma \in S_{4}$ and we introduced a rotation matrix $\hat{\rho}_{\sigma}$ by acting with $\sigma$ on the prefactor of 
eq. (2.14), i.e.

$$
\begin{aligned}
\hat{\rho}_{\sigma}\left(x_{i}\right) & =\rho_{\sigma(1) \sigma(2)}\left(x_{\sigma(1)}, x_{\sigma(2)}\right) \otimes \rho_{\sigma(3) \sigma(4)}\left(x_{\sigma(3)}, x_{\sigma(4)}\right) \\
\text { where } \rho_{i j}\left(x_{i}, x_{j}\right) & =\pi_{i}(e) \otimes \pi_{j}\left(s_{x_{i j}} s_{e_{d}}\right) .
\end{aligned}
$$

Throughout most of this work we shall work with the $s$-channel. Formulas for other channels can be obtained easily by acting on labels with the appropriate permutations.

Let us conclude this section with a few more comments on eq. (2.14). In order to appreciate the details a bit better, it is useful to think of the argument of the function $F_{s}$ as a product $g=g_{l} g_{r}^{-1}$ of two elements

$$
g_{l}=e^{I x_{21} \cdot K} e^{x_{1} \cdot P}, \quad g_{r}=e^{I x_{43} \cdot K} e^{x_{3} \cdot P} .
$$

Both $g_{l}$ and $g_{r}$ are elements of the conformal group $G=\operatorname{Spin}(1, d+1)$, though of a quite special form. Since they are generated from translations and special conformal transformations only, they can be thought of as parametrizing the coset space $K \backslash G$ in which rotations and dilations are trivial. Thereby, the coset space $K \backslash G$ may be considered as the configuration space of a pair of points in the conformal compactification of the Euclidean space. There exists an intimate relation with embedding space constructions. Recall that a point in Euclidean space is represented by a light-like line in embedding space $\mathbb{R}^{1, d+1}$. Hence, any two points in Euclidean space determine a pair of light-like lines and hence a 2-dimensional plane $P \subset \mathbb{R}^{1, d+1}$ in embedding space. For the first two points $x_{1}$ and $x_{2}$ the corresponding plane $P_{x_{1}, x_{2}}$ is actually encoded in the element $g_{l}$. More concretely, if we represent $g_{l}$ in the $d+2$-dimensional (fundamental) representation of the conformal group $G$, the first two rows of this matrix - which consist of a light-like and a time-like vector in a $d+2$ - dimensional Minkowski space - span the plane $P_{x_{1}, x_{2}}$ in embedding space. The remaining $d$ rows, span the orthogonal complement $P_{x_{1}, x_{2}}^{\perp}$ in embedding space. A change of basis in 2-dimensional subspace $P$ and its orthogonal complement $P^{\perp}$ is mediated by left multiplication with elements $k_{l} \in K=\mathrm{SO}(1,1) \times \mathrm{Spin}(d)$. The action of conformal transformations on the pair of points $x_{1}$ and $x_{2}$, on the other hand, is given by the right action of group $G$ on the coset $K \backslash G$. All this is true for the other two insertion points $x_{3}, x_{4}$ and the group element $g_{r}$ we construct from these two points. The argument $g_{l} g_{r}^{-1}$ of our function $F_{s}$ in eq. (2.14) therefore admits a left action of $K_{l} \cong K$ and a right action of $K_{r} \cong K$. The right action of the conformal group on $g_{l} \in K \backslash G$ and the left action on $g_{r}^{-1} \in G / K$ are inverse to each other so that the element $g_{l} g_{r}^{-1} \in K \backslash G / K$ is conformally invariant. Note that the double coset $K \backslash G / K$ is 2-dimensional, in agreement with the fact that one can form two independent conformal invariants from four points in $\mathbb{R}^{d}$. Since our functions $F_{s}$ satisfy the covariance law (2.13) under the action of $K_{l} \times K_{r}$, the section $F_{s}\left(g_{l} g_{r}^{-1}\right)$ is entirely determined by the values it takes on the two-dimensional space of conformal invariants, just as it is the case for conformal correlators $G$. These comments may help to appreciate the content of the main result (2.14) in this section.

\section{Cartan coordinates and tensor structures}

As it stands the formula (2.14) may not seem very useful. All we have done was to express a function $G$ satisfying a set of covariance laws, the conformal Ward identities, in terms 
of a $K_{l} \times K_{r}$ covariant function $F$ on the conformal group. In this section, we will use the Cartan decomposition of the conformal group to solve the $K_{l} \times K_{r}$ covariance laws. Geometric properties of this decomposition ensure that the Casimir equations take the form of a matrix Calogero-Sutherland system.

As described in the introduction our analysis will allow us to decompose the correlation function $G$ into a product of some scalar function $\tilde{\Omega}$, the tensor structures $\Theta$ and some function $\psi$ of two variables $u_{1}$ and $u_{2}$. The choice of these coordinates and the form of the associated Casimir equations will be reviewed briefly in the first part before we turn to the other two factors in the second subsection. We will determine $\tilde{\Omega}$ completely and from first principles and explain how the tensor structures $\Theta$ are computed, leaving fully explicit formulas to the subsequent sections.

\subsection{Cartan coordinates and Calogero-Sutherland models}

In order to evaluate the right hand side of equation (2.14) further it is advantageous to change coordinates and work with a coordinate system that arises from the KAK or Cartan decomposition of the conformal group, as explained in [20,21]. The Cartan coordinates generalize the usual Euler angles for $\mathrm{SO}(3)$ to the $d$-dimensional conformal group in a way that is ideally adapted to the covariance law (2.13) we discussed in the previous section.

The Cartan decomposition is based on the decomposition of the Lie algebra

$$
\mathfrak{g}=\mathfrak{k} \oplus \mathfrak{p},
$$

where $\mathfrak{k}=\operatorname{Lie}(K)$ and $\mathfrak{p}$ is its orthogonal complement with respect to the Killing form. The latter is spanned by translations $P_{\mu}$ and special conformal transformations $K_{\mu}, \mu=1, \ldots, d$. Let us select a 2-dimensional abelian subalgebra $\mathfrak{a}$ of $\mathfrak{p}$ that is spanned by $A_{+}=\left(P_{1}+K_{1}\right) / 2$ and $A_{-}=\left(P_{2}-K_{2}\right) / 2$. Through exponentiation we pass to the abelian subgroup $A \subset$ $\operatorname{Spin}(1, d+1)$ that consists of elements of the form

$$
a\left(u_{1}, u_{2}\right)=e^{\frac{u_{1}+u_{2}}{2} A_{+}-i \frac{u_{1}-u_{2}}{2} A_{-}}=e^{\frac{u_{1}+u_{2}}{4}\left(P_{1}+K_{1}\right)-i \frac{u_{1}-u_{2}}{4}\left(P_{2}-K_{2}\right)} .
$$

Almost all elements ${ }^{2}$ of the conformal group can be factorized as

$$
g=k_{l} a\left(u_{1}, u_{2}\right) k_{r}
$$

with $k_{l}, k_{r} \in K$. This factorization is not unique, however, since elements of $A$ commute with $b \in B=\operatorname{Spin}(d-2) \subset \operatorname{Spin}(d)$. Consequently, the group element $g$ is invariant under the action $\left(k_{l}, k_{r}\right) \mapsto\left(k_{l} b, b^{-1} k_{r}\right)$ of $\operatorname{Spin}(d)$ on $K \times K$. We can fix this gauge freedom by choosing a section $k_{l} \in K / B$.

Let us now insert the Cartan decomposition (3.2) into the right hand side of our representation (2.15) of the correlation function $G$. Using the covariance law (2.13) we see that

$$
F_{s}(g)=\hat{\pi}_{s}\left(k_{l}, k_{r}\right) f_{s}\left(u_{1}, u_{2}\right)
$$

\footnotetext{
${ }^{2}$ We use this standard mathematical terminology which means "all elements up to a Haar measure zero subset".
} 
where $f_{s}\left(u_{1}, u_{2}\right)$ denotes the restriction of $F_{s}$ from the conformal group $G$ to the elements (3.1) of the torus $A \subset G$. The map $\hat{\pi}_{s}$ was defined in eq. (2.11). The factorization (3.3) of the function $F_{s}$ into a matrix $\hat{\pi}$ on $K_{l} \times K_{r}$ and a function $f_{s}$ of two variables is key to our discussion in this section. Most of this work will focus on the matrix $\hat{\pi}$. The function $f_{s}$, on the other hand, was analysed in much detail in [20] and [21]. We will use the rest of this subsection to review the results and in particular precise relation of $f$ with Calogero-Sutherland wave functions which we mentioned above.

In order to explain the mathematical nature of $f$ we start from the Laplacian $\Delta_{G}^{(2)}$ on the conformal group. From the start, $\Delta_{G}^{(2)}$ acts as a second order differential operator on functions $F$ which are defined for any $g \in G$. By construction, however, it is invariant under both left and right actions of the conformal group on itself and hence under the action of $K_{l} \cong K$ and $K_{r} \cong K$, in particular. Consequently, the Laplacian descends to a second order differential operator on the space of covariant functions $\Gamma_{\sigma}^{\pi_{i}}$. Due to the Cartan decomposition, all functions in this space are determined by their restrictions to the subgroup $A$. Since the decomposition suffers from the ambiguity of shifting elements $b \in B=\operatorname{Spin}(d-2)$ between $k_{l}$ and $k_{r}$, the function $f$ can only take values in a subspace $W_{P}^{B} \subset W_{P}$ of $B$ invariants in the space $W_{P}$ of all polarizations. The action of the Laplacian on the restriction $f$ may be worked out explicitly and contains both second and first order derivatives with respect to the two coordinates $u_{1}$ and $u_{2}$. Thanks to the fact that the Cartan decomposition is hyperpolar [21], one can eliminate the first order terms through a simple transformation of the form ${ }^{3}$

$$
\begin{aligned}
f_{s}\left(u_{1}, u_{2}\right) & =\omega^{-1 / 2}\left(u_{1}, u_{2}\right) \psi_{s}\left(u_{1}, u_{2}\right) \\
\text { where } \omega\left(u_{1}, u_{2}\right) & =4(-1)^{2-d}\left(\sinh \frac{u_{1}}{2} \sinh \frac{u_{2}}{2}\right)^{2 d-2} \operatorname{coth} \frac{u_{1}}{2} \operatorname{coth} \frac{u_{2}}{2}\left|\sinh ^{-2} \frac{u_{1}}{2}-\sinh ^{-2} \frac{u_{2}}{2}\right|^{d-2} .
\end{aligned}
$$

Putting eqs. (3.3) and (3.4) together we can write

$$
F_{s}(g)=\Xi_{s}^{\hat{\pi}}(g) \psi_{s}\left(u_{1}, u_{2}\right) \quad \text { where } \Xi_{s}^{\hat{\pi}}(g)=\omega\left(u_{1}, u_{2}\right)^{-1 / 2} \hat{\pi}_{s}\left(k_{l}, k_{r}\right): W_{P}^{B} \mapsto W_{P} .
$$

The product $\omega \hat{\pi}$ certainly acts on the entire space $W_{P}$ of polarizations. On the other hand, $f_{s}$ only takes values in the $B=\operatorname{Spin}(d-2)$ invariant subspace of tensor structures. For this reason we want to think of $\Xi$ as a map from tensor structures to polarizations. Our discussion about the relation between the Laplacian $\Delta_{G}^{(2)}$ on the conformal group $G$ and Calogero-Sutherland Hamiltians $H^{\mathrm{CS}}$ can now be written as

$$
\Delta_{G}^{(2)} \Xi_{s}^{\hat{\pi}}(g)=\Xi_{s}^{\hat{\pi}}(g)\left[\frac{1}{2} H_{\hat{\pi}, s}^{\mathrm{CS}}+\frac{1}{8}\left(d^{2}-2 d+1\right)\right] .
$$

Here $\Delta_{G}$ is a second order differential operator in all $(d+2)(d+1) / 2$ coordinates on the $d$ dimensional conformal group $G=\operatorname{Spin}(1, d+1)$. The whole equation is valid when applied to functions of two coordinates $u_{1}$ and $u_{2}$. The Hamiltonian $H^{\mathrm{CS}}$ takes the form

$$
H_{\hat{\pi}, s}^{\mathrm{CS}}=-\frac{\partial^{2}}{\partial u_{1}^{2}}-\frac{\partial^{2}}{\partial u_{2}^{2}}+V_{\hat{\pi}, s}^{\mathrm{CS}}\left(u_{1}, u_{2}\right)
$$

\footnotetext{
${ }^{3}$ The numerical prefactor here is fixed from matching normalization of Dolan-Osborn for the case of scalar blocks [25] with the standard normalization of the Harish-Chandra wavefunctions in Calogero-Sutherland theory.
} 
where $V$ is a matrix valued potential that depends on the representation theoretic data $\pi_{i}, i=1, \ldots, 4$. We shall see a few examples below. In the case all the four external fields are scalar, i.e. $\lambda_{i}=0$, the potential is scalar as well and reads

$$
\begin{aligned}
& V_{\hat{\pi}, s}^{\mathrm{CS}}\left(u_{i}\right)=V_{(a, b, \epsilon)}^{\mathrm{CS}}\left(u_{i}\right)=V_{(a, b)}^{P T}\left(u_{1}\right)+V_{(a, b)}^{P T}\left(u_{2}\right)+\frac{\epsilon(\epsilon-2)}{8 \sinh ^{2} \frac{u_{1}-u_{2}}{2}}+\frac{\epsilon(\epsilon-2)}{8 \sinh ^{2} \frac{u_{1}+u_{2}}{2}}, \\
& V_{(a, b)}^{P T}(u)=\frac{(a+b)^{2}-\frac{1}{4}}{\sinh ^{2} u}-\frac{a b}{\sinh ^{2} \frac{u}{2}} .
\end{aligned}
$$

Here the three parameters $a, b, \epsilon$ are given by $2 a=\Delta_{2}-\Delta_{1}, 2 b=\Delta_{3}-\Delta_{4}$ and $\epsilon=d-2$. The derivation of eq. (3.5) along with the computation of the potentials for a number of examples can be found in [20,21, 26].

\subsection{Cartan decomposition and tensor structures}

After this digression into Casimir equations and Calogero-Sutherland models let us now come back to the study of the correlation function $G$ and in particular the matrix $\hat{\pi}$ in the factorization (3.3). In order to make further progress we need to study the Cartan decomposition (3.2) of the elements $g\left(x_{i}\right)$ in the argument of the function $F_{s}$ and in particular how it depends in the insertion points $x_{i}$,

$$
g\left(x_{i}\right)=e^{I x_{21} \cdot K} e^{x_{13} \cdot P} e^{I x_{34} \cdot K}=k_{l}\left(x_{i}\right) a\left(x_{i}\right) k_{r}\left(x_{i}\right) .
$$

We will work out explicit formulas for the dependence of $k_{l}$ and $k_{r}$ on the insertion points of the fields in $d=3,4$ dimensions below. For the moment let us content ourselves with stating results for the factor $a\left(x_{i}\right)$ as well as the dilations $d_{l}, d_{r}$ in $k_{l}=d_{l} r_{l}$ and $k_{r}=d_{r} r_{r}$. It is not too difficult to show, see appendix $\mathrm{C}$, that the variables $u_{i}$ which parametrize elements $a$ depend on $x_{i}$ as

$$
\begin{aligned}
e^{u_{i}} & =1-\frac{2}{z_{i}}\left(1+\sqrt{1-z_{i}}\right) \\
\text { where } \quad z_{1} z_{2} & =\frac{x_{12}^{2} x_{34}^{2}}{x_{13}^{2} x_{24}^{2}}, \quad\left(1-z_{1}\right)\left(1-z_{2}\right)=\frac{x_{14}^{2} x_{23}^{2}}{x_{13}^{2} x_{24}^{2}} .
\end{aligned}
$$

As we suggested above, the coordinates $u_{i}$, are directly related to the cross ratios. In fact, their exponentials are identical to the radial coordinates of [27]. The relation (3.9) had already been observed on a case-by-case basis in [18, 20,21]. Our analysis here provides a proper derivation for all four-point blocks, both scalar and spinning.

Similarly it is not difficult to find an explicit relation between the insertion points $x_{i}$ and the coordinates $\lambda_{l}$ and $\lambda_{r}$ on the dilation subgroup

$$
d_{l}=d\left(\lambda_{l}\right)=e^{\lambda_{l} D}, \quad d_{r}=d\left(\lambda_{r}\right)=e^{\lambda_{r} D}
$$

with

$$
e^{2 \lambda_{l}}=\frac{x_{12}^{2} x_{14}^{2}}{x_{24}^{2}} \frac{1}{\sqrt{\left(1-z_{1}\right)\left(1-z_{2}\right)}}, \quad e^{2 \lambda_{r}}=\frac{x_{14}^{2}}{x_{13}^{2} x_{34}^{2}} \frac{1}{\sqrt{\left(1-z_{1}\right)\left(1-z_{2}\right)}}
$$


The derivation of these relatively simple formulas for $\lambda_{l}$ and $\lambda_{r}$ as well as the derivation of eq. (3.9) can be found in appendix C. In summary we have shown that the correlation function $G$ defined in eq. (2.7) takes the form

$$
\begin{aligned}
G\left(x_{i}\right) & =\frac{1}{x_{12}^{2 \Delta_{2}} x_{34}^{2 \Delta_{4}}} e^{2 a \lambda_{l}} e^{2 b \lambda_{r}} \Theta_{s}\left(x_{i}\right) F_{s}\left(a\left(u_{1}, u_{2}\right)\right) \\
& =\frac{1}{x_{12}^{\Delta_{1}+\Delta_{2}} x_{34}^{\Delta_{3}+\Delta_{4}}}\left(\frac{x_{14}}{x_{24}}\right)^{2 a}\left(\frac{x_{14}}{x_{13}}\right)^{2 b}\left[\left(1-z_{1}\right)\left(1-z_{2}\right)\right]^{-\frac{a}{2}-\frac{b}{2}} \Theta_{s}\left(x_{i}\right) f_{s}\left(u_{1}, u_{2}\right) \\
& =\frac{\frac{1}{2}(-1)^{\frac{d-2}{2}}}{x_{12}^{\Delta_{1}+\Delta_{2}} x_{34}^{\Delta_{3}+\Delta_{4}}}\left(\frac{x_{14}}{x_{24}}\right)^{2 a}\left(\frac{x_{14}}{x_{13}}\right)^{2 b} \frac{\left(z_{1} z_{2}\right)^{\frac{d-1}{2}}\left|z_{1}-z_{2}\right|^{-\frac{d-2}{2}}}{\left[\left(1-z_{1}\right)\left(1-z_{2}\right)\right]^{\frac{a}{2}+\frac{b}{2}+\frac{1}{4}}} \cdot \Theta_{s}\left(x_{i}\right) \cdot \psi_{s}\left(u_{1}, u_{2}\right)
\end{aligned}
$$

where

$$
\Theta_{s}\left(x_{i}\right)=\hat{\rho}_{s}\left(x_{i}\right) \hat{\pi}_{s}\left(r_{l}\left(x_{i}\right), r_{r}\left(x_{i}\right)\right): W_{P}^{B} \mapsto W_{P}
$$

is a map from the space $W_{P}^{B}$ of tensor structures to the space $W_{P}$ of polarisations, see also our discussion above. The rotation maps $\hat{\rho}_{s}$ and $\hat{\pi}_{s}$ were defined in eqs. (2.16) and (2.11). Recall that the index $s$ stands for $s$-channel and corresponds to the unit element $\sigma=e \in S_{4}$ of the permutation group.

Formula (3.15) is the central equation of this work. It describes the relation between four-point correlators and Calogero-Sutherland wave functions for arbitrary fields with spin and is reminiscent of more standard decompositions of four-point functions. Our decomposition of the function $G\left(x_{i}\right)$ contains three basic ingredients which we separated by · in eq. (3.15). The first part is a function of the insertion points which differs from the function

$$
\Omega\left(x_{i}\right)=\frac{1}{x_{12}^{\Delta_{1}+\Delta_{2}} x_{34}^{\Delta_{3}+\Delta_{4}}}\left(\frac{x_{14}}{x_{24}}\right)^{2 a}\left(\frac{x_{14}}{x_{13}}\right)^{2 b}
$$

in the work of Dolan and Osborn by a specific function of the cross ratios. The second part are the tensor structures $\Theta$, i.e. a set of maps from the space of tensor structures to the space of polarizations. In contrast to other approaches, we are able to provide a universal construction through eq. (3.16) that allows us to construct $\Theta$ for any spin assignment through the evaluation of certain rotation matrices. We will illustrate the usefulness of this construction in the next sections. The final ingredient is the Calogero-Sutherland wave function $\psi$ which takes values in the space of four-point tensor structures and replaces the blocks $g=\left(g^{I}\right)$ in more conventional approaches. The functions $g^{I}(z, \bar{z})$ satisfy Casimir equations which have been worked out in a number of examples, but depend very much on specific choices, in particular on the basis of tensor structures. On the other hand, with our specific choice of the other two parts, i.e. the function $\tilde{\Omega}$ and the tensor structures $\Theta$, the Casimir equations for $\psi$ are guaranteed to take the form of a Calogero-Sutherland eigenvalue equation.

After these comments on the formula (3.15) let us work out one example right away, namely the case of scalar blocks for which the spaces $V_{i} \cong \mathbb{C}$ carry a trivial representation 
of the rotation group and hence $\Theta_{s}$ is the identity map. In this case we find

$$
\begin{aligned}
G\left(x_{i}\right) & =\frac{1}{x_{12}^{\Delta_{1}+\Delta_{2}} x_{34}^{\Delta_{3}+\Delta_{4}}}\left(\frac{x_{14}}{x_{24}}\right)^{2 a}\left(\frac{x_{14}}{x_{13}}\right)^{2 b}\left[\left(1-z_{1}\right)\left(1-z_{2}\right)\right]^{-\frac{a}{2}-\frac{b}{2}} f_{s}\left(u_{1}, u_{2}\right) \\
& =\frac{1}{x_{12}^{\Delta_{1}+\Delta_{2}} x_{34}^{\Delta_{3}+\Delta_{4}}}\left(\frac{x_{14}}{x_{24}}\right)^{2 a}\left(\frac{x_{14}}{x_{13}}\right)^{2 b} \cdot \frac{(-1)^{\frac{d-2}{2}}}{2} \frac{\left(z_{1} z_{2}\right)^{\frac{d-1}{2}}\left|z_{1}-z_{2}\right|^{-\frac{d-2}{2}}}{\left[\left(1-z_{1}\right)\left(1-z_{2}\right)\right]^{\frac{d}{2}+\frac{b}{2}+\frac{1}{4}}} \cdot \psi_{s}\left(u_{1}, u_{2}\right) .
\end{aligned}
$$

Here we have written the result as a product of three factors which are separated by a $\cdot$ The first factor coincides with the function (3.17) that Dolan and Osborn used to write the scalar four-point function $G$ as $G=\Omega g$ in terms of a function $g=g(z, \bar{z})$ of the cross ratios. The second factor denotes the gauge transformation that was introduced in [18] to relate blocks $g$ with Calogero-Sutherland wave functions $\psi$. The final factor $\psi$ is related to the functions $f_{s}\left(u_{1}, u_{2}\right)$ in the previous line through eq. (3.4). Thereby we have now fully derived the relation between Calogero-Sutherland models and scalar four-point correlators using group theory methods alone.

\section{Computation of tensor structures in $d=3,4$}

In the previous section we discussed how to compute the tensor structures $\Theta$ from the Cartan decomposition (3.8) of the group elements $g\left(x_{i}\right)$. The resulting formula (3.16) for $\Theta$ through representation matrices of the elements $r_{l}$ and $r_{r}$ may still seem a bit abstract, but it is not that difficult to work with. The precise details depend on the dimension $d$ (or rather the value of $d \bmod 8$ ) with dimensions $d=2 n-1$ and $d=2 n$ being closely related. The first such pair $d=1$ and $d=2$ is of course trivial since all irreducible representations of the rotation group are one-dimensional. We will therefore illustrate our formulas with the first non-trivial pair of dimensions $d=3$ and $d=4$. In both cases, the conformal group possesses a 4-dimensional representation which we will employ to perform the Cartan decomposition. We begin with a few general steps that are common to both examples before we specialize to $d=3$ dimensions in the second subsection and $d=4$ in the third.

\subsection{Spinor representation of the conformal group}

In the two cases $d=3$ and $d=4$, the smallest non-trivial representation of the complexified conformal algebra is 4-dimensional. Our central task is to decompose the elements $g\left(x_{i}\right)$ of the conformal group as prescribed in eq. (3.8). We can perform this factorization in any faithful representation, including the 4-dimensional one. For the 4-dimensional conformal group, the 4-dimensional representation is related to the realization in terms of $\operatorname{SL}(2, \mathbb{H})$, see also appendix D. The 3 -dimensional conformal group $\operatorname{Spin}(1,4)$ is a subgroup of $\operatorname{Spin}(1,5)$ and hence we can pass from the latter to the former by restricting the range of the Greek indices $\mu, \nu=1, \ldots, d$. These indices are raised and lowered by the flat Euclidean metric $g_{\mu \nu}=\delta_{\mu \nu}$. 
In the 4-dimensional representation, we consider a basis in which the generators of dilations, translations and special conformal transformations read,

$$
D=\frac{1}{2}\left(\begin{array}{cc}
I & 0 \\
0 & -I
\end{array}\right), \quad P_{\mu}=\left(\begin{array}{cc}
0 & \sigma_{\mu} \\
0 & 0
\end{array}\right), \quad K_{\mu}=\left(\begin{array}{cc}
0 & 0 \\
\bar{\sigma}_{\mu} & 0
\end{array}\right) .
$$

All these matrices are written in terms of $2 \times 2$ block matrices, with $I$ denoting the $2 \times 2$ identity matrix. The matrices $\sigma_{\mu}$ and $\bar{\sigma}_{\mu}$ are

$$
\sigma_{\mu}=\left(-\sigma_{3},-i I, \sigma_{1},-\sigma_{2}\right), \quad \bar{\sigma}_{\mu}=\left(-\sigma_{3}, i I, \sigma_{1},-\sigma_{2}\right) .
$$

They are chosen such that in particular

$$
\operatorname{det}(a \cdot \sigma)=\operatorname{det}\left(a_{\mu} \sigma^{\mu}\right)=-a_{\mu} a^{\mu}=-\|a\|^{2} .
$$

With these conventions, the following relations are readily seen to hold

$$
e^{a^{\mu} P_{\mu}}=\left(\begin{array}{cc}
1 & a^{\mu} \sigma_{\mu} \\
0 & 1
\end{array}\right), \quad e^{a^{\mu} K_{\mu}}=\left(\begin{array}{cc}
1 & 0 \\
a^{\mu} \bar{\sigma}_{\mu} & 1
\end{array}\right) .
$$

These formulas make it easy to work out the group elements $g\left(x_{i}\right)$ that are defined on the left hand side of eq. (3.8). In the representation under consideration, these elements become

$$
g=e^{I x_{21} \cdot K} e^{x_{13} \cdot P} e^{I x_{34} \cdot K}=\left(\begin{array}{cc}
1+y_{13} \bar{y}_{34} & y_{13} \\
\bar{y}_{21}+\bar{y}_{34}+\bar{y}_{21} y_{13} \bar{y}_{34} & 1+\bar{y}_{21} y_{13}
\end{array}\right),
$$

where we have introduced

$$
\bar{y}_{21}=I x_{21}^{\mu} \bar{\sigma}_{\mu}, \quad y_{13}=x_{13}^{\mu} \sigma_{\mu}, \quad \bar{y}_{34}=I x_{34}^{\mu} \bar{\sigma}_{\mu} .
$$

This is the matrix we want to decompose into a product $k_{l} a k_{r}$. As before we split elements $k \in K$ explicitly into dilations and rotations, i.e. we factorize $g$ as

$$
g=e^{\lambda_{l} D} r_{l} a r_{r} e^{\lambda_{r} D} .
$$

In the 4-dimensional representation and with our notation using $2 \times 2$ matrices, the decomposition takes the form

$$
g\left(x_{i}\right)=\left(\begin{array}{cc}
e^{\frac{1}{2} \lambda_{l}} & 0 \\
0 & e^{-\frac{1}{2} \lambda_{l}}
\end{array}\right) r_{l}\left(x_{i}\right)\left(\begin{array}{cc}
C & S \\
S & C
\end{array}\right) r_{r}\left(x_{i}\right)\left(\begin{array}{cc}
e^{\frac{1}{2} \lambda_{r}} & 0 \\
0 & e^{-\frac{1}{2} \lambda_{r}}
\end{array}\right),
$$

where $\exp \left(\lambda_{l} / 2\right)$ should be read as a $2 \times 2$ matrix block, i.e. it is $\exp \left(\lambda_{l} / 2\right) \mathbf{1}_{2}$ etc. The matrix in the middle is the 4-dimensional representation of the factor $a\left(x_{i}\right)$. Its blocks are given by

$$
C=\left(\begin{array}{cc}
\cosh \frac{u_{1}}{2} & 0 \\
0 & \cosh \frac{u_{2}}{2}
\end{array}\right), \quad S=\left(\begin{array}{cc}
-\sinh \frac{u_{1}}{2} & 0 \\
0 & \sinh \frac{u_{2}}{2}
\end{array}\right)
$$


With our choice of the dilation generator, the rotation matrices $r_{l}$ and $r_{r}$ are block diagonal, i.e. they read

$$
r_{l}\left(x_{i}\right)=\left(\begin{array}{cc}
L\left(x_{i}\right) & 0 \\
0 & L^{\prime}\left(x_{i}\right)
\end{array}\right), \quad r_{r}\left(x_{i}\right)=\left(\begin{array}{cc}
R\left(x_{i}\right) & 0 \\
0 & R^{\prime}\left(x_{i}\right)
\end{array}\right),
$$

where $L, L^{\prime}, R, R^{\prime}$ are $2 \times 2$ rotation matrices with unit determinant. We note that all but the middle factor $a(x)$ in the decomposition of $g$ are block diagonal. Consequently, we can extract the coordinates $\lambda_{i}$ and $u_{i}$ from $g$ by considering determinants of the $2 \times 2$ blocks. Equating the four possible $2 \times 2$ subdeterminants in eqs. (4.1) and (4.2) yields the following relations

$$
e^{4 \lambda_{l}}=\frac{x_{12}^{4} x_{13}^{2} x_{14}^{2}}{x_{23}^{2} x_{24}^{2}}, \quad e^{4 \lambda_{r}}=\frac{x_{14}^{2} x_{24}^{2}}{x_{13}^{2} x_{23}^{2} x_{34}^{4}},
$$

and

$$
\cosh ^{2} \frac{u_{1}}{2} \cosh ^{2} \frac{u_{2}}{2}=\frac{x_{14}^{2} x_{23}^{2}}{x_{12}^{2} x_{34}^{2}}, \quad \sinh ^{2} \frac{u_{1}}{2} \sinh ^{2} \frac{u_{2}}{2}=\frac{x_{13}^{2} x_{24}^{2}}{x_{12}^{2} x_{34}^{2}},
$$

from which we recover the results (3.9) and (3.12) we stated in the previous section. Our main goal, however is to determine $r_{l}$ and $r_{r}$ now. After moving the dilations from left hand side of eq. (4.2) to the right we obtain

$$
\left(\begin{array}{ll}
L\left(x_{i}\right) C R\left(x_{i}\right) & L\left(x_{i}\right) S R^{\prime}\left(x_{i}\right) \\
L^{\prime}\left(x_{i}\right) S R\left(x_{i}\right) & L^{\prime}\left(x_{i}\right) C R^{\prime}\left(x_{i}\right)
\end{array}\right)=\left(\begin{array}{cc}
e^{-\frac{\lambda_{l}+\lambda_{r}}{2}}\left(1+y_{13} \bar{y}_{34}\right) & e^{-\frac{\lambda_{l}-\lambda_{r}}{2}} y_{13} \\
e^{\frac{\lambda_{l}-\lambda_{r}}{2}}\left(\bar{y}_{21}+\bar{y}_{34}+\bar{y}_{21} y_{13} \bar{y}_{34}\right) & e^{\frac{\lambda_{l}+\lambda_{r}}{2}}\left(1+\bar{y}_{21} y_{13}\right)
\end{array}\right) .
$$

The rest of our analysis is case dependent and will be carried out separately for $d=3$ and $d=4$ in the following two subsections.

\subsection{3-dimensional spinning blocks}

Calculating $\Theta_{s}$ is in principle straightforward, if only a bit tedious. Rotation matrices in the 3-dimensional rotation group are parametrized by three Euler angles and since the stabilizer subgroup $B=\operatorname{Spin}(d-2)$ is trivial for $d=3$, we need to determine six angles in terms of the insertion points $x_{i}$ by solving the equation (4.6). To be more precise, let us denote the generators of the Lie algebra $\mathfrak{s u}(2)$ by $\left\{M_{12}, M_{13}, M_{23}\right\}$. On the group we use the Euler angles $(\phi, \theta, \psi)$ such that

$$
r(\phi, \theta, \psi)=e^{-\phi M_{12}} e^{-\theta M_{23}} e^{-\psi M_{12}} .
$$

In the 4-dimensional representation the generators $M_{i j}$ are represented by

$$
M_{23}=-\frac{i}{2} \operatorname{diag}\left(\sigma_{1},-\sigma_{1}\right), \quad M_{13}=-\frac{i}{2} \operatorname{diag}\left(\sigma_{2}, \sigma_{2}\right), \quad M_{12}=-\frac{i}{2} \operatorname{diag}\left(\sigma_{3},-\sigma_{3}\right) .
$$

When these matrices are inserted into eq. (4.7), we obtain the matrices $r_{l}=r_{l}\left(\phi_{l}, \theta_{l}, \psi_{l}\right)$ and $r_{r}=r_{r}\left(\phi_{r}, \theta_{r}, \psi_{r}\right)$. The $2 \times 2$ matrix block $L$, for example, is given by

$$
L=\left(\begin{array}{cc}
\cos \frac{\theta_{l}}{2} e^{i \frac{\phi_{l}+\psi_{l}}{2}} & i \sin \frac{\theta_{l}}{2} e^{i \frac{\phi_{l}-\psi_{l}}{2}} \\
i \sin \frac{\theta_{l}}{2} e^{-i \frac{\phi_{l}-\psi_{l}}{2}} & \cos \frac{\theta_{l}}{2} e^{-i \frac{\phi_{l}+\psi_{l}}{2}}
\end{array}\right) .
$$


and similarly for the matrix block $R$, but with angles carrying the subscript $r$ instead of $l$. The blocks $L^{\prime}$ and $R^{\prime}$ are computed similarly. Note that the right hand side of eq. (4.6) is written in terms of the $x_{i}$ already and so are the matrix elements $C$ and $S$. Hence, eq. (4.6) indeed provides us with eight equations that allow to determine the six unknown angles in terms of $x_{i}$. Once these angles are known, we can evaluate eq. (4.7) in any representation and thereby compute $\hat{\pi}_{s}\left(r_{l}, r_{r}\right)$ for fields of arbitrary spin.

This may sound difficult, but it can be carried out quite efficiently. We will first see how it is done for seed blocks, i.e. when $r_{l}$ and $r_{r}$ are represented in the 2-dimensional fundamental representation of $\mathfrak{s u}(2)$. Then we employ some elementary $\mathrm{SU}(2)$ representation theory to find $\pi_{s}\left(r_{l}, r_{r}\right)$ for higher dimensional spin representations.

\subsubsection{Seed conformal blocks}

Let us now turn to the first example of a spinning correlator and determine its tensor structure $\Theta_{s}$. The correlation function we want to look at,

$$
G^{b}{ }_{a}\left(x_{i}\right)=\left\langle\psi^{\Delta_{1}}\left(x_{1}\right)^{b} \phi^{\Delta_{2}}\left(x_{2}\right) \phi^{\Delta_{3}}\left(x_{2}\right) \psi^{\Delta_{4}}\left(x_{4}\right)_{a}\right\rangle
$$

involves two scalar fields at $x_{2}$ and $x_{3}$ of weight $\Delta_{2}$ and $\Delta_{3}$, respectively, along with two fermionic fields of spin $\lambda_{1} / 2=\lambda_{4} / 2=1 / 2$ and weight $\Delta_{1}$ and $\Delta_{4}$. In this case, the spaces $V_{2}$ and $V_{3}$ are 1-dimensional while $V_{1}$ and $V_{4}$ are 2-dimensional (vectors in the spin-1/2 representation carry a Latin index $a, b \ldots)$. Hence, the space $W_{P}$ of polarizations has 4 basis elements, as does the space $W_{P}^{B}=W_{P}$ of tensor structures.

For this setup we now want to compute the tensor structures $\Theta_{s}$. According to eq. (3.16), the $4 \times 4$ matrix $\Theta_{s}$ is a product of the matrix $\hat{\rho}_{s}\left(x_{i}\right)$ defined in eq. (2.16) and the matrix $\hat{\pi}$ which we introduced in eq. (2.11). The former is very simple to calculate. Since $\pi_{2}$ is trivial and $\pi_{4}$ is spin- $1 / 2$ representation, $\hat{\rho}_{s}$ reads

$$
\hat{\rho}_{s}\left(x_{i}\right)=\frac{1}{\left|x_{34}\right|}\left(\begin{array}{ll}
1 & 0 \\
0 & 1
\end{array}\right) \otimes\left(\begin{array}{cc}
x_{34}^{3} & -x_{34}^{1}-i x_{34}^{2} \\
x_{34}^{1}-i x_{34}^{2} & x_{34}^{3}
\end{array}\right) .
$$

Calculating $\hat{\pi}$ is a bit more of a challenge. According to eq. $(2.11), \hat{\pi}=\pi_{12}\left(r_{l}\right) \otimes \pi_{34}\left(r_{r}^{-1}\right)$ is the tensor product of the $2 \times 2$ matrices

$$
\begin{aligned}
\pi_{12}\left(r_{l}\right) & =\left(\begin{array}{cc}
\cos \frac{\theta_{l}}{2} e^{i \frac{\phi_{l}+\psi_{l}}{2}} & i \sin \frac{\theta_{l}}{2} e^{i \frac{\phi_{l}-\psi_{l}}{2}} \\
i \sin \frac{\theta_{l}}{2} e^{-i \frac{\phi_{l}-\psi_{l}}{2}} & \cos \frac{\theta_{l}}{2} e^{-i \frac{\phi_{l}+\psi_{l}}{2}}
\end{array}\right), \\
\pi_{34}\left(r_{r}^{-1}\right) & =\left(\begin{array}{cc}
\cos \frac{\theta_{r}}{2} e^{i \frac{\phi_{r}+\psi_{r}}{2}} & -i \sin \frac{\theta_{r}}{2} e^{i \frac{\phi_{r}-\psi_{r}}{2}} \\
-i \sin \frac{\theta_{r}}{2} e^{-i \frac{\phi_{r}-\psi_{r}}{2}} & \cos \frac{\theta_{r}}{2} e^{-i \frac{\phi_{r}+\psi_{r}}{2}}
\end{array}\right)^{-1} .
\end{aligned}
$$

Through an explicit computation one may show that equation (4.1) is equivalent to the following equation for $\hat{\pi}$,

$$
\hat{\pi}_{s}\left(r_{l}, r_{r}\right)=p(g) D_{1}^{-1} p(a)^{-1}
$$

Here, $p$ is the following linear map on the space of $4 \times 4$ matrices $M$,

$$
p(M)=\left(\begin{array}{cccc}
-M_{13} & M_{12} & -M_{43} & -M_{42} \\
M_{14} & M_{11} & M_{44} & -M_{41} \\
-M_{23} & M_{22} & M_{33} & M_{32} \\
M_{24} & M_{21} & -M_{34} & M_{31}
\end{array}\right)
$$


On the right hand side of eq. (4.14) it acts on 4-dimensional representation matrices $g$ of elements in the conformal group as well as on the special elements $a$ whose building blocks were stated in eq. (4.3). The matrix $D_{1}$, finally, is the diagonal matrix

$$
D_{1}=\operatorname{diag}\left(e^{\frac{\lambda_{1}-\lambda_{2}}{2}}, e^{\frac{\lambda_{1}+\lambda_{2}}{2}}, e^{\frac{-\lambda_{1}-\lambda_{2}}{2}}, e^{\frac{-\lambda_{1}+\lambda_{2}}{2}}\right) .
$$

To prove equation (4.14), one only needs to calculate both sides. The simplicity of the relation follows from the fact that in the 4-dimensional representation of the conformal group, rotations are represented essentially as in the spin- $1 / 2$ representation of $\mathrm{SU}(2)$ in terms of Pauli matrices. The identity holds true for any element that admits a Cartan decomposition. The definition of the map $p$ may come a bit ad hoc, but it has a nice group theoretic origin that is sketched in appendix D.

So far we thought of $\hat{\pi}$ as a function of six angles. But, the equation (4.14) effectively represents it in terms of the insertion points $x_{i}$ of our four fields. To this end, we simply insert the special elements $g$ which are constructed from the insertion points as stated in eq. (4.1). Similarly, the matrix elements of $a$ are determined by the insertion points through the cross ratios $u_{i}$, as given in eq. (4.3). When written in terms of the insertion points through eq. (4.4), the diagonal matrix $D_{1}$ reads

$$
D_{1}=\operatorname{diag}\left(\sqrt{\frac{x_{12} x_{13} x_{34}}{x_{24}}}, \sqrt{\frac{x_{12} x_{14}}{x_{23} x_{34}}}, \sqrt{\frac{x_{23} x_{34}}{x_{12} x_{14}}}, \sqrt{\frac{x_{24}}{x_{12} x_{13} x_{34}}}\right) .
$$

This completes the construction of $\hat{\pi}$ and hence, along with the expression (4.11) for $\hat{\rho}_{s}$, of the tensor structures $\Theta_{s}$ for seed blocks in $d=3$ dimensions.

For completeness let us also briefly review the form of the corresponding Casimir equations. For seed blocks in the Calogero-Sutherland gauge these were studied in [20]. The Hamiltonian has block-diagonal ${ }^{4}$ form $H=\operatorname{diag}\left\{H_{1}, H_{2}\right\}$ with the following potentials

$$
\begin{aligned}
& \tilde{V}_{1}^{\mathrm{CS}}\left(u_{1}, u_{2}\right)=\left(\begin{array}{cc}
V_{(a, b, 1)}^{\mathrm{CS}}\left(u_{1}, u_{2}\right) & 0 \\
0 & V_{(a, b, 1)}^{\mathrm{CS}}\left(u_{1}, u_{2}\right)
\end{array}\right)+ \\
& \left(\begin{array}{cc}
\frac{-1}{16}\left(\frac{1}{\cosh ^{2} \frac{u_{1}}{2}}+\frac{1}{\cosh ^{2} \frac{u_{2}}{2}}+\frac{2}{\cosh ^{2} \frac{u_{1}-u_{2}}{4}}+\frac{2}{\cosh ^{2} \frac{u_{1}+u_{2}}{4}}\right) & \frac{a+b}{4}\left(\frac{1}{\cosh ^{2} \frac{u_{1}}{2}}-\frac{1}{\cosh ^{2} \frac{u_{2}}{2}}\right) \\
\frac{a+b}{4}\left(\frac{1}{\cosh ^{2} \frac{u_{1}}{2}}-\frac{1}{\cosh ^{2} \frac{u_{2}}{2}}\right) & \frac{-1}{16}\left(\frac{1}{\cosh ^{2} \frac{u_{1}}{2}}+\frac{1}{\cosh ^{2} \frac{u_{2}}{2}}-\frac{2}{\sinh ^{2} \frac{u_{1}-u_{2}}{4}}-\frac{2}{\sinh ^{2} \frac{u_{1}+u_{2}}{4}}\right)
\end{array}\right) \\
& \tilde{V}_{2}^{\mathrm{CS}}\left(u_{1}, u_{2}\right)=\left(\begin{array}{cc}
V_{(a, b, 1)}^{\mathrm{CS}}\left(u_{1}, u_{2}\right) & 0 \\
0 & V_{(a, b, 1)}^{\mathrm{CS}}\left(u_{1}, u_{2}\right)
\end{array}\right)+ \\
& \left(\begin{array}{cc}
\frac{1}{16}\left(\frac{1}{\sinh ^{2} \frac{u_{1}}{2}}+\frac{1}{\sinh ^{2} \frac{u_{2}}{2}}+\frac{2}{\sinh ^{2} \frac{u_{1}+u_{2}}{4}}-\frac{2}{\cosh ^{2} \frac{u_{1}-u_{2}}{4}}\right) & \frac{b-a}{4}\left(\frac{1}{\sinh ^{2} \frac{u_{1}}{2}}-\frac{1}{\sinh ^{2} \frac{u_{2}}{2}}\right) \\
\frac{b-a}{4}\left(\frac{1}{\sinh ^{2} \frac{u_{1}}{2}}-\frac{1}{\sinh ^{2} \frac{u_{2}}{2}}\right) & \frac{1}{16}\left(\frac{1}{\sinh ^{2} \frac{u_{1}}{2}}+\frac{1}{\sinh ^{2} \frac{u_{2}}{2}}+\frac{2}{\sinh ^{2} \frac{u_{1}-u_{2}}{4}}-\frac{2}{\cosh ^{2} \frac{u_{1}+u_{2}}{4}}\right)
\end{array}\right)
\end{aligned}
$$

where $V_{(a, b, 1)}^{\mathrm{CS}}$ is a Calogero-Sutherland Hamiltonian, see eq. (3.6) and the $\tilde{V}$ differs from $V$ by a constant gauge transformation of the form

$$
\tilde{V}_{i}^{\mathrm{CS}}=u V_{i}^{\mathrm{CS}} u^{-1} \quad \text { with } \quad u=\frac{1}{\sqrt{2}}\left(\begin{array}{cc}
1 & 1 \\
-1 & 1
\end{array}\right) .
$$

\footnotetext{
${ }^{4}$ In the basis we use throughout this work, the first block matrix $H_{1}$ actually appears in the second and third row/column while $\mathrm{H}_{2}$ acts on the subspace spanned by the first and fourth basis vector.
} 
Eigenfunctions of this Hamiltonian provide us with the $s$-channel wave functions $\psi_{s}\left(u_{1}, u_{2}\right)$ in eq. (3.15). These eigenfunctions were mapped to conformal blocks in [20]. In order to derive the transformation between blocks and Calogero-Sutherland wave functions from group theory, we can now compare eq. (3.15) with the expansion in tensor structures that was found in [16]. This comparison will be carried out in the next section and we will see that the results are in complete agreement.

\subsubsection{Tensor structures for arbitrary spins}

The tensor structures for seed blocks we constructed in the previous subsection, and in particular the factor $\hat{\pi}$ given in eq. (4.14), was so simple because $\hat{\pi}$ contains the same combinations of left and right Euler angles as the matrix elements on the left hand side of eq. (4.6). When dealing with more general spinning fields, we need to construct the left and right Euler angles separately in terms of the insertion points. This is possible, but the formulas are not quite as elegant as eq. (4.14). In fact, departing from $4 \times 4$ matrix we defined in eq. (4.14) we can reconstruct the individual factors defined in eqs. (4.12) and (4.13) as

$$
\pi_{12}\left(r_{l}\right)=\left(\begin{array}{cc}
\sqrt{\frac{\varpi_{33}}{\varpi_{11}}\left(\varpi_{11} \varpi_{44}-\varpi_{12} \varpi_{43}\right)} & i \sqrt{\frac{\varpi_{13}}{\varpi_{31}}\left(1-\varpi_{11} \varpi_{44}+\varpi_{12} \varpi_{43}\right)} \\
i \sqrt{\frac{\varpi_{31}}{\varpi_{13}}\left(1-\varpi_{11} \varpi_{44}+\varpi_{12} \varpi_{43}\right)} & \sqrt{\frac{\varpi_{11}}{\varpi_{33}}\left(\varpi_{11} \varpi_{44}-\varpi_{12} \varpi_{43}\right)}
\end{array}\right),
$$

and

$$
\pi_{34}\left(r_{r}^{-1}\right)=\left(\begin{array}{cc}
\sqrt{\frac{\varpi_{22}}{\varpi_{11}}\left(\varpi_{22} \varpi_{33}-\varpi_{13} \varpi_{42}\right)} & i \sqrt{\frac{\varpi_{12}}{\varpi_{21}\left(1-\varpi_{22} \varpi_{33}+\varpi_{13} \varpi_{42}\right)}} \\
i \sqrt{\frac{\varpi_{21}}{\varpi_{12}}\left(1-\varpi_{22} \varpi_{33}+\varpi_{13} \varpi_{42}\right)} & \sqrt{\frac{\varpi_{11}}{\varpi_{22}}\left(\varpi_{22} \varpi_{33}-\varpi_{13} \varpi_{42}\right)}
\end{array}\right) .
$$

Here $\varpi\left(x_{i}\right)=\hat{\pi}^{-1}$ denotes the inverse of the matrix $\hat{\pi}$ we constructed in eq. (4.14). Note that the matrix elements of $\varpi$ and hence the matrix entries of the two $2 \times 2$ matrices $\pi_{12}\left(r_{l}\right)$ and $\pi_{34}\left(r_{r}^{-1}\right)$ are functions of the insertion points $x_{i}$.

Having constructed the factors $\pi_{12}\left(r_{l}\right)$ and $\pi_{34}\left(r_{r}^{-1}\right)$ for fields in the fundamental 2dimensional representation of $\operatorname{Spin}(3)$, we can now obtain these factors for all other representations with the help of some standard group theory constructions. To fix notations let us recall that the spin- $l$ representation of $\mathfrak{s u}(2)$ is spanned by the basis vectors $\{|l, m\rangle, m=-l,-l+1, \ldots, l\}$. By definition, these are eigenvectors of $M_{12}$, i.e. they obey $M_{12}|l, m\rangle=-i m|l, m\rangle$. Matrix elements in the spin- $l$ representation are given by

$$
t_{m n}^{l}(\phi, \theta, \psi)=\langle l, m|g(\phi, \theta, \psi)| l, n\rangle=e^{-i(m \phi+n \psi)} d_{m n}^{l}(\theta),
$$

where the Wigner $d$-function $d_{m n}^{l}$ is expressed in terms of Jacobi polynomials as

$$
d_{m n}^{l}(\theta)=i^{m-n} \sqrt{\frac{(l+m) !(l-m) !}{(l+n) !(l-n) !}}\left(\sin \frac{\theta}{2}\right)^{m-n}\left(\cos \frac{\theta}{2}\right)^{m+n} P_{l-m}^{(m-n, m+n)}(\cos \theta) .
$$

In particular, the spin-1/2 (fundamental) representation takes the form

$$
\tau(\phi, \theta, \psi)=\left(\begin{array}{cc}
\cos \frac{\theta}{2} e^{i \frac{\phi+\psi}{2}} & i \sin \frac{\theta}{2} e^{i \frac{\phi-\psi}{2}} \\
i \sin \frac{\theta}{2} e^{-i \frac{\phi-\psi}{2}} & \cos \frac{\theta}{2} e^{-i \frac{\phi+\psi}{2}}
\end{array}\right) .
$$


From the matrix elements of the fundamental representation, we can obtain matrix elements of any other irreducible representation as

$$
t_{m n}^{l}(g)=(-1)^{m-n} \sqrt{\frac{(l+m) !(l-m) !}{(l+n) !(l-n) !}} \tau_{22}^{m+n} \tau_{21}^{m-n} P_{l-m}^{(m-n, m+n)}\left(\tau_{11} \tau_{22}+\tau_{12} \tau_{21}\right) .
$$

Suppose now that we want to find the tensor structures for correlators in which we insert a field of spin $l$ at $x_{1}$, while keeping the field at $x_{2}$ to be scalar. Then the corresponding matrix factor $\pi_{12}^{l}$ of $\hat{\pi}$ can be obtained by inserting the matrix elements of the matrix defined in eq. (4.19) into the previous formula. Other spin assignments may be dealt with similarly and hence, our equations (4.19), (4.20) and (4.23) completely solve the problem of constructing tensor structures for all $d=3$ dimensional spinning conformal blocks in the Calogero-Sutherland gauge.

\subsection{4-dimensional spinning seed blocks}

Our goal in this subsection is to construct the tensor structures for the first non-trivial seed blocks in $d=4$ dimensions. In order to do so we can follow the steps we described in the previous subsection. In the 4-dimensional theory, we need to determine 11 Euler angles in total. The rotation group Spin(4) itself is 6-dimensional so that left and right rotations together are parametrized by 12 angles, but one of these angles is redundant because of the non-trivial stabilizer subgroup $\operatorname{Spin}(d-2)=\operatorname{Spin}(2)$. To be specific, we parametrize the left and right rotations $r_{l}$ and $r_{r}$ as

$$
r_{l}=e^{\varphi_{1}^{l} X_{1}} e^{\theta_{1}^{l} Z_{1}} e^{\psi_{1}^{l} X_{1}} e^{\varphi_{2}^{l} X_{2}} e^{\theta_{2}^{l} Z_{2}} e^{\psi_{2}^{l} X_{2}}, \quad r_{r}=e^{\varphi_{1}^{r} X_{1}} e^{\theta_{1}^{r} Z_{1}} e^{\psi_{1}^{r} X_{1}} e^{\varphi_{2}^{r} X_{2}} e^{\theta_{2}^{r} Z_{2}} e^{\psi_{2}^{r} X_{2}} .
$$

In order to reduce down to 11 angles we impose the additional condition $\psi_{2}^{l}=-\psi_{1}^{l}$. The symbols $X_{i}$ and $Z_{i}, i=1,2$ denote the following linear combinations of the rotation matrices $M_{i j}$

$X_{1}=-\frac{1}{2}\left(M_{12}+M_{34}\right), \quad X_{2}=\frac{1}{2}\left(M_{12}-M_{34}\right), \quad Z_{1}=-\frac{1}{2}\left(M_{14}+M_{23}\right), \quad Z_{2}=\frac{1}{2}\left(M_{14}-M_{23}\right)$.

The $4 \times 4$ matrices $M_{i j}$ in turn possess a simple representation in terms of Pauli matrices. For $M_{12}, M_{23}$ and $M_{13}$ this was spelled out already in our discussion of the 3-dimensional theory, see eqs. (4.8). The remaining matrices $M_{i j}$ are given by

$$
M_{14}=-\frac{i}{2} \operatorname{diag}\left(\sigma_{1}, \sigma_{1}\right), \quad M_{24}=-\frac{i}{2} \operatorname{diag}\left(-\sigma_{2}, \sigma_{2}\right), \quad M_{34}=-\frac{i}{2} \operatorname{diag}\left(\sigma_{3}, \sigma_{3}\right) .
$$

Following [21] we shall study a set of correlators that involve the non-trivial 4-dimensional seed blocks of [17]. These turn out to appear in the decomposition of

$$
G_{\dot{a}}^{b}\left(x_{i}\right)=\left\langle\Phi_{0,0}\left(x_{1}\right) \Phi_{s, 0}\left(x_{2}\right) \Phi_{0,0}\left(x_{3}\right) \Phi_{0, s}\left(x_{4}\right)\right\rangle,
$$

where $s \in(0,1 / 2,1, \ldots)$. Labels $\left(s_{1}, s_{2}\right)=\left(j_{1}, j_{2}\right)$ that we attached to the operators $\Phi_{s_{1}, s_{2}}$ refer to the representation of the rotation group. We consider the case with $s=$ $1 / 2$. The corresponding representations $V_{2}$ and $V_{4}$ are then both 2-dimensional. Their 
vectors are written with undotted and dotted Latin indices, respectively. The space $W_{P}$ of polarizations has dimension four and the space of $B=\operatorname{Spin}(2)$-invariants is 2-dimensional.

In order to construct the map $\Theta_{s}$ we need to compute both $\hat{\rho}_{s}$ and $\hat{\pi}$. The former is given by

$$
\hat{\rho}_{s}\left(x_{i}\right)=\frac{1}{\left|x_{12}\right|\left|x_{34}\right|}\left(\begin{array}{cc}
x_{21}^{4}-i x_{21}^{3} & x_{21}^{2}-i x_{21}^{1} \\
-x_{21}^{2}-i x_{21}^{1} & x_{21}^{4}+i x_{21}^{3}
\end{array}\right) \otimes\left(\begin{array}{cc}
x_{34}^{4}-i x_{34}^{3} & x_{34}^{2}+i x_{34}^{1} \\
-x_{34}^{2}+i x_{34}^{1} & x_{34}^{4}+i x_{34}^{3}
\end{array}\right) .
$$

This is obtained by evaluating the rotation matrix $s_{x} s_{e_{d}}$ in the 2-dimensional representations $(1 / 2,0)$ and $(0,1 / 2)$ of the rotation group, see eq. (2.16). Calculating $\hat{\pi}$ is a bit more involved. With Euler angles introduced as described above, the left and right representations of the rotation generators are given by

$$
\begin{aligned}
& \pi_{12}\left(r_{l}\right)=\left(\begin{array}{ll}
\cos \frac{\theta_{2}^{l}}{2} e^{-i \frac{\phi_{2}^{l}+\psi_{2}^{l}}{2}} & i \sin \frac{\theta_{2}^{l}}{2} e^{-i \frac{\phi_{2}^{l}-\psi_{2}^{l}}{2}} \\
i \sin \frac{\theta_{2}^{l}}{2} e^{i \frac{\phi_{2}^{l}-\psi_{2}^{l}}{2}} & \cos \frac{\theta_{2}^{l}}{2} e^{i \frac{\phi_{2}^{l}+\psi_{2}^{l}}{2}}
\end{array}\right), \\
& \pi_{34}\left(r_{r}\right)=\left(\begin{array}{ll}
\cos \frac{\theta_{1}^{r}}{2} e^{-i \frac{\phi_{1}^{r}+\psi_{1}^{r}}{2}} & i \sin \frac{\theta_{1}^{r}}{2} e^{-i \frac{\phi_{1}^{r}-\psi_{1}^{r}}{2}} \\
i \sin \frac{\theta_{1}^{r}}{2} e^{i \frac{\phi_{1}^{r}-\psi_{1}^{r}}{2}} & \cos \frac{\theta_{1}^{r}}{2} e^{i \frac{\phi_{1}^{r}+\psi_{1}^{r}}{2}}
\end{array}\right),
\end{aligned}
$$

where $\psi_{2}^{l}=-\psi_{1}^{l}$. We need to calculate the tensor product $\hat{\pi}$ of these two matrices as a function of the insertion points $x_{i}$ to obtain the main building block for the desired tensor structure. As in the previous section the resulting expression is surprisingly simple. It requires to introduce the following linear map $q: M_{2 \times 2} \times M_{2 \times 2} \rightarrow M_{2 \times 4}$ that sends a pair of $2 \times 2$ matrices $M, N$ to a rectangular $2 \times 4$ matrix of the form

$$
q(M, N)=\left(\begin{array}{l}
r(M) \\
r(N)
\end{array}\right), \quad r(M)=\left(\begin{array}{llll}
M_{12} & M_{11} & M_{22} & M_{21}
\end{array}\right) .
$$

From the four $2 \times 2$ matrix blocks of the group element $g$ in the 4 -dimensional representation we can construct a pair of $2 \times 2$ matrices $M=M_{2}$ and $N=M_{1}$ as

$$
M_{i}=\sinh ^{2} \frac{u_{i}}{2} D B^{-1} A-\cosh ^{2} \frac{u_{i}}{2} C=\sinh ^{2} \frac{u_{i}}{2} y_{13}^{-1}-\bar{y}_{21}-\bar{y}_{34}-\bar{y}_{21} y_{13} \bar{y}_{34} .
$$

The second equality applies to special elements $g\left(x_{i}\right)$ that we assigned to a set of insertion points in eq. (4.1). In terms of these two matrices one can now compute $\hat{\pi}$ as

$$
\hat{\pi}\left(r_{l}, r_{r}\right)^{-1}=\mathcal{P}\left(\pi_{12}\left(r_{l}\right) \otimes \pi_{34}\left(r_{r}^{-1}\right)\right)^{-1}=\frac{2 e^{\frac{\lambda_{1}-\lambda_{2}}{2}}}{\cosh u_{1}-\cosh u_{2}}\left(\begin{array}{cc}
\sinh \frac{u_{1}}{2} & 0 \\
0 & \sinh \frac{u_{2}}{2}
\end{array}\right) q\left(M_{2}, M_{1}\right) .
$$

Here, $\mathcal{P}$ is the projector to the space of $B$-invariants

$$
\mathcal{P}=\left(\begin{array}{llll}
0 & 1 & 0 & 0 \\
0 & 0 & 1 & 0
\end{array}\right),
$$

see [21] for its derivation. Note that the matrices $\hat{\pi}$ and $\hat{\pi}^{-1}$ are not square. When we invert the $2 \times 4$ matrix $\hat{\pi}^{-1}$ defined in eq. (4.28) we are instructed to build a $4 \times 2$ matrix $\hat{\pi}$ such that

$$
\hat{\pi}\left(r_{l}, r_{r}\right)^{-1} \hat{\pi}\left(r_{l}, r_{r}\right)=I_{2}
$$


Again, the identity (4.28) holds for any element of the conformal group that has a Cartan decomposition, as can be checked by calculating both sides. The application to $g\left(x_{i}\right)$ is obtained by substituting eq. (4.27) into eq. (4.28).

The Calogero-Sutherland potential for the case under consideration was constructed in [21], and reads

$$
\begin{aligned}
& \tilde{V}_{\frac{1}{2}}^{\mathrm{CS}}\left(u_{1}, u_{2}\right)=\left(\begin{array}{cc}
V_{(a, b, 2)}^{\mathrm{CS}}\left(u_{1}, u_{2}\right)-\frac{1}{8} & 0 \\
0 & V_{(a, b, 2)}^{\mathrm{CS}}\left(u_{1}, u_{2}\right)-\frac{1}{8}
\end{array}\right)+ \\
& \frac{1}{16}\left(\begin{array}{cc}
\frac{1}{\sinh ^{2} \frac{u_{1}}{2}}+\frac{1}{\sinh ^{2} \frac{u_{2}}{2}}+\frac{4}{\sinh ^{2} \frac{u_{1}-u_{2}}{4}}-\frac{4}{\cosh ^{2} \frac{u_{1}+u_{2}}{4}} & 4(b-a)\left(\frac{1}{\sinh ^{2} \frac{u_{1}}{2}}-\frac{1}{\sinh ^{2} \frac{u_{2}}{2}}\right) \\
4(b-a)\left(\frac{1}{\sinh ^{2} \frac{u_{1}}{2}}-\frac{1}{\sinh ^{2} \frac{u_{2}}{2}}\right) & \frac{1}{\sinh ^{2} \frac{u_{1}}{2}}+\frac{1}{\sinh ^{2} \frac{u_{2}}{2}}+\frac{4}{\sinh ^{2} \frac{u_{1}+u_{2}}{4}}-\frac{4}{\cosh ^{2} \frac{u_{1}-u_{2}}{4}}
\end{array}\right) .
\end{aligned}
$$

where once again $\tilde{V}$ is related to the potential $V$ by the same constant gauge transformation $u$ we introduced in eq. (4.18).

The tensor structures $\Theta_{s} \sim \hat{\rho}_{s} \hat{\pi}$ we computed above resembles the tensor structures constructed in [17]. In [21] a map $\chi$ was found that sends the conformal blocks of [17] to the Calogero-Sutherland wave functions $\psi_{s}$. One may show that the composition of this map with the tensor structures of [17] reproduces the tensor structures $\Theta_{s}$ we have constructed in this subsection, see next section.

Recall that in the 3-dimensional theory we discussed how to obtain tensor structures for any spin assignments in the correlator. The construction proceeded in two steps. First we factorized the construction of $\hat{\pi}$ in eq. (4.14) for the seed block into explicit expressions for the fundamental left and right matrices in terms of insertion points, see eqs. (4.19) and (4.20). Then, in a second step, we used standard SU(2) group theory to build left and right representation matrices in any other representation. Here we could do something very similar except that the information encoded in eq. (4.28) is not sufficient to reconstruct all 11 Euler angles and hence to reconstruct the left and right rotation matrices. In fact, the linear map $\hat{\pi}$ given in eq. (4.28) only contains $2 \times 4=8$ matrix elements and hence we do not have enough equations to solve for the 11 angles. In order to obtain the missing equations one needs to compute $\hat{\pi}$ for one other spin assignment. Once the left and right rotations are fixed in terms of the insertion points of the four fields, one can proceed as in the 3-dimensional case since the 4-dimensional rotation group $\mathrm{SO}(4)$ is a double cover of $\mathrm{SO}(3) \times \mathrm{SO}(3)$. Hence representation matrices in higher spin representations can be computed from those in the two fundamental representations in the same way as we explained at the end of the previous subsection. So, in principle it is not much more complicated to obtain tensor structures for arbitrary spin assignments in $d=4$ dimensions.

\section{Comparison with other approaches}

In this section, we want to compare our tensor structures in Calogero-Sutherland gauge with the ones that were constructed in the literature previously, most notably in [16] on 3-dimensional seed blocks and in [17] for the 4-dimensional case. Conformal blocks in these two different gauges had previously been related in [20] and [21], respectively, through a 
comparison of the relevant Casimir equations. Now that we have a systematic theory of tensor structures for the Calogero-Sutherland gauge, it is possible to also compare the tensor structures with the ones in the literature, at least for the cases that have been worked out. It is a nice consistency check to see that the results match.

In conventional approaches to spinning conformal blocks, one chooses some more or less natural or simple set of tensor structures for each case at hand, i.e. a given assignment of spins, and then expands the correlator in these tensor structures,

$$
G^{a}\left(\pi_{i} ; x_{i}\right)=\Omega\left(\Delta_{i} ; x_{i}\right) \sum_{I} t_{I}^{a}\left(x_{i}\right) g^{I}\left(\pi_{i} ; z, \bar{z}\right) .
$$

Here, $\Omega$ is the usual scalar function (3.17) that was introduced by Dolan and Osborn, the superscript $a$ runs through a basis of polarizations and $I$ labels some basis of tensor structures. We shall think of $T=\left(t_{I}^{a}\right)$ as a matrix of tensor structures. As in the text above, it maps the space of tensor structures to the space of polarizations. The tensor structures $T$ are chosen such that the functions $g^{I}(z, \bar{z})$ depend on cross ratios only. Once the tensor structures are fixed, one can derive Casimir equations for the associated conformal blocks $g^{I}$. Of course the form of the Casimir equations depends on the choice of the tensor structures.

In contrast to this standard theory, our construction of tensor structures $\Theta_{s}$ is systematic and canonical. This implies that the Casimir equations are canonical as well, i.e. they always come out to be of Calogero-Sutherland form. The decomposition of spinning correlators in terms of Calogero-Sutherland wave functions was given in eq. (1.1). This should be compared with the decomposition (5.1), i.e. symbolically

$$
\Omega T \chi=\tilde{\Omega} \Theta
$$

where $\chi=\left(\chi_{J}^{I}\right)$ is a transformation in the space of tensor structures that relates the blocks $g^{I}$ to the Calogero Sutherland wave functions $\psi^{J}$. We will now compute this map $\chi$ for seed blocks in $d=3$ and $d=4$ dimensions and compare with the results in [20] and [21]. Throughout this section we shall omit the subscript $s$ since all quantities are in the $s$-channel.

\section{$5.1 \quad 3$-dimensional spinning blocks}

Let us begin the discussion of the 3-dimensional case by reviewing the construction of tensor structures for the only non-trivial seed blocks in [16]. The constructions in that work are performed in the Minkowski space with the metric

$$
g_{\mu \nu}=\operatorname{diag}(-1,1,1) .
$$

Greek indices $\mu, \nu \ldots$ are raised and lowered with this metric. Greek indices $\alpha, \beta \ldots$ from the beginning of the alphabet are raised and lowered with the $\operatorname{Sp}(2)$ symplectic form according to

$$
\Omega_{\alpha \beta}=\left(\begin{array}{cc}
0 & 1 \\
-1 & 0
\end{array}\right), \quad \psi_{\alpha}=\Omega_{\alpha \beta} \psi^{\beta}
$$


A vector $x^{\mu}$ is made into a $2 \times 2$ matrix with the help of 3-dimensional gamma matrices

$$
\left(\gamma^{\mu}\right)_{\beta}^{\alpha}=\left(i \sigma_{2}, \sigma_{1}, \sigma_{3}\right),
$$

where $\sigma_{i}$ are the Pauli matrices. We write

$$
x_{\beta}^{\alpha}=x_{\mu}\left(\gamma^{\mu}\right)_{\beta}^{\alpha} .
$$

The correlation function (4.10) of two scalar and two spinor fields then assumes the form

$$
\left\langle\psi_{1}^{\alpha}\left(x_{1}\right) \varphi_{2}\left(x_{2}\right) \varphi_{3}\left(x_{3}\right) \psi_{4}^{\beta}\left(x_{4}\right)\right\rangle=\Omega\left(x_{i}\right) \sum_{I=1}^{4} t_{I}^{\alpha \beta} g^{I}(z, \bar{z}),
$$

where the index $I=1, \ldots, 4$ runs over the following four-point tensor structures

$$
\begin{aligned}
t_{1}^{\alpha \beta} & =i \frac{\left(x_{14} i \sigma_{2}\right)^{\alpha \beta}}{\left|x_{14}\right|}, & t_{2}^{\alpha \beta} & =-i \frac{\left(x_{12} x_{23} x_{34} i \sigma_{2}\right)^{\alpha \beta}}{\left|x_{12}\right|\left|x_{23}\right|\left|x_{34}\right|}, \\
t_{3}^{\alpha \beta} & =i \frac{\left(x_{12} x_{24} i \sigma_{2}\right)^{\alpha \beta}}{\left|x_{12}\right|\left|x_{24}\right|}, & t_{4}^{\alpha \beta} & =i \frac{\left(x_{13} x_{34} i \sigma_{2}\right)^{\alpha \beta}}{\left|x_{13}\right|\left|x_{34}\right|},
\end{aligned}
$$

and $\Omega\left(x_{i}\right)$ is the function (3.17) introduced by Dolan and Osborn. The matrix $T=\left(t_{I}^{\alpha \beta}\right)$ is the most nontrivial factor that relates the correlation function $G$ with the conformal blocks $g^{I}$. It bears some resemblance with the tensor structures $\Theta=\Theta_{s}$ we computed in section 4.2 , and most notably with the matrix $p\left(g\left(x_{i}\right)\right)$ in eq. (4.14). More precisely one can see that

$$
c T \chi=\hat{\rho} p(g) D_{1}^{-1} p(a)^{-1}
$$

where $c=\Omega / \tilde{\Omega}$ and the matrix $\chi$ takes the form

$$
\chi=\left(\begin{array}{cccc}
0 & A\left(\chi_{1}-\chi_{2}\right) & A\left(-\chi_{1}-\chi_{2}\right) & 0 \\
0 & A\left(-\chi_{1}-\chi_{2}\right) & A\left(\chi_{1}-\chi_{2}\right) & 0 \\
B\left(\chi_{3}-\chi_{4}\right) & 0 & 0 & B\left(\chi_{3}+\chi_{4}\right) \\
B\left(-\chi_{3}-\chi_{4}\right) & 0 & 0 & B\left(-\chi_{3}+\chi_{4}\right)
\end{array}\right)
$$

with $A=-i / 2$ and $B=-1 / 2 .{ }^{5}$ The functions $\chi_{i}\left(u_{1}, u_{2}\right)$ are defined in (A.10) of [20], with $x=u_{1}, y=u_{2}$. Let us note that in checking eq. (5.4) it is advantageous to bring the factor $p(a)$ to the left hand side and use

$$
\operatorname{c\chi p}\left(a\left(x_{i}\right)\right)=\left(\begin{array}{cccc}
0 & \sqrt{\frac{x_{14} x_{23}}{x_{12} x_{34}}} & 0 & 0 \\
0 & 0 & \sqrt{\frac{x_{14} x_{23}}{x_{12} x_{34}}} & 0 \\
0 & 0 & 0 & \sqrt{\frac{x_{13} x_{24}}{x_{12} x_{34}}} \\
\sqrt{\frac{x_{13} x_{24}}{x_{12} x_{34}}} & 0 & 0 & 0
\end{array}\right) .
$$

This concludes our discussion of tensor structures for 3-dimensional seed blocks. The tensor structures for other blocks have not been stated in the literature.

\footnotetext{
${ }^{5}$ The comparison of Casimir equations that was used in [20] to determine $\chi$ does not determine the numerical factors $A$ and $B$. The map that was spelled out in that work corresponds to $A=1 / \sqrt{2}=B$.
} 


\subsection{4-dimensional spinning blocks}

Let us begin the discussion of the 4-dimensional case by reviewing the construction of tensor structures for seed blocks in $[17,28]$. It is set in Minkowski signature with the metric

$$
g_{\mu \nu}=\operatorname{diag}(-1,1,1,1) .
$$

Greek indices from the second half of the alphabet are raised and lowered with this metric $g$. Indices $\alpha, \beta \ldots$ from the beginning of the Greek alphabet label the basis of the 2 -dimensional representation $(1 / 2,0)$ of $\mathrm{SO}(1,3)$. Similarly, the dotted indices $\dot{\alpha}, \dot{\beta} \ldots$ enumerate a basis in the representation $(0,1 / 2)$. These are raised and lowered with the Levi-Civita symbol according to

$$
\psi_{\alpha}=\varepsilon_{\alpha \beta} \psi^{\beta}, \quad \varepsilon_{\alpha \beta}=\left(\begin{array}{cc}
0 & -1 \\
1 & 0
\end{array}\right)
$$

The same formulas hold for the dotted indices. The vector representation is equivalent to the tensor product $(1 / 2,0) \otimes(0,1 / 2)$. This equivalence can be realised explicitly with the help of $\sigma$-matrices

$$
x_{\alpha \dot{\beta}}=x_{\mu} \sigma_{\alpha \dot{\beta}}^{\mu}, \quad \sigma_{\alpha \dot{\beta}}^{\mu}=\left(-I, \sigma^{i}\right) .
$$

Further we write $\left(\bar{\sigma}^{\mu}\right)^{\dot{\alpha} \beta}=\left(-I,-\sigma^{i}\right)$ and define the corresponding $\bar{x}^{\dot{\alpha} \beta}$ in the obvious way.

With this preparation let us now look at the correlation function (4.25). It decomposes over the tensor structures introduced above as ${ }^{6}$

$$
G^{\dot{\alpha} \beta}\left(x_{i}\right)=\mathcal{K}_{4}\left(x_{i}\right) \sum_{I} T_{I}^{\dot{\alpha} \beta}\left(x_{i}\right) g^{I}(U, V)=\mathcal{K}_{4}\left(x_{i}\right) \sum_{e=0}^{2 s} g_{e}^{(2 s)}(U, V) I_{42}^{e} J_{42,31}^{p-e},
$$

where the scalar prefactor $\mathcal{K}_{4}$ is defined by

$$
\mathcal{K}_{4}=\Omega \sqrt{\frac{x_{13}}{x_{12} x_{24} x_{34}}}
$$

and the tensors $I, J$ on the right hand side take the form

$$
\begin{aligned}
I_{i j}^{\dot{\alpha} \beta} & =x_{i j}^{\mu} \bar{\sigma}_{\mu}^{\dot{\alpha} \beta}, \\
J_{i j, k l}^{\dot{\alpha} \beta} & =\frac{-1}{x_{k l}^{2}}\left(\left[x_{i k}, x_{j l}\right]^{\mu}+\left[x_{j k}, x_{i l}\right]^{\mu}+\left[x_{i j}, x_{l k}\right]^{\mu}-2 i \varepsilon^{\mu \nu \rho \sigma} x_{i k \nu} x_{l j \rho} x_{l k \sigma}\right) \bar{\sigma}_{\mu}^{\dot{\alpha} \beta},
\end{aligned}
$$

with $[x, y]^{\mu}=x^{2} y^{\mu}-y^{2} x^{\mu}$. The second tensor can be written more compactly as

$$
J_{i j, k l}^{\dot{\alpha} \beta}=\frac{2}{x_{k l}^{2}} \bar{x}_{i k}^{\dot{\alpha} \gamma}\left(x_{k l}\right)_{\gamma \dot{\delta}} \bar{x}_{l j}^{\dot{\delta} \beta} .
$$

The case we analysed in section 4.3 corresponds to $2 s=p=1$ and hence the correlation function has the form

$$
G^{\dot{\alpha} \beta}\left(x_{i}\right)=\mathcal{K}_{4}\left(x_{i}\right)\left(g_{0}^{(1)}(U, V) J_{42,31}^{\dot{\alpha} \beta}+g_{1}^{(1)}(U, V) I_{42}^{\dot{\alpha} \beta}\right),
$$

\footnotetext{
${ }^{6}$ Following the conventions in [17] we label seed blocks by an integer $p=2 s$ rather than the spin $s$ itself.
} 
involving two tensor structures, in agreement with the dimension of the space $W_{P}^{B}$ of tensor structures in our analysis.

In order to compare the tensor structures from [17] with our tensor structures given in eqs. (4.26) and (4.28) we note that the two discussions of the seed correlators use a different basis in the space of polarizations. Comparing the conventions in [28] and [21] one can see that the basis transformation is mediated by the following matrix

$$
M_{\dot{a} \dot{\alpha}}^{b \beta}=\frac{i}{2}\left(\begin{array}{cccc}
1 & 1 & -1 & -1 \\
-1 & -1 & -1 & -1 \\
1 & -1 & -1 & 1 \\
-1 & 1 & -1 & 1
\end{array}\right) .
$$

Taking this necessary change of basis into account we have to prove that

$$
M \Omega T \chi=\tilde{\Omega} \Theta .
$$

It is not difficult to check that this equation is indeed satisfied with $\chi$ given by

$$
\chi=\frac{\left(\operatorname{coth} \frac{u_{1}}{2} \operatorname{coth} \frac{u_{2}}{2}\right)^{-a-b-\frac{1}{2}}}{\left(\cosh u_{1}-\cosh u_{2}\right)^{2}}\left(\begin{array}{cc}
\sinh \frac{u_{1}}{2} & \sinh \frac{u_{2}}{2} \\
2 \sinh ^{-1} \frac{u_{1}}{2} & 2 \sinh ^{-1} \frac{u_{2}}{2}
\end{array}\right) .
$$

This coincides with the map between Calogero-Sutherland eigenfunctions and conformal blocks that was found in [21] based on the comparison of the Casimir differential equations. The easiest way to verify eq. (5.5) passes through the following formula

$$
c \chi \hat{\pi}^{-1}=\frac{e^{\frac{\lambda_{1}-\lambda_{2}}{2}} \sinh \frac{u_{1}}{2} \sinh \frac{u_{2}}{2}}{\left(\cosh u_{1}-\cosh u_{2}\right)^{2}}\left(\begin{array}{c}
2 \sinh ^{2} \frac{u_{1}}{2} \sinh ^{2} \frac{u_{2}}{2} r_{1}-\left(\sinh ^{2} \frac{u_{1}}{2}+\sinh ^{2} \frac{u_{2}}{2}\right) r_{2} \\
2\left(\sinh ^{2} \frac{u_{1}}{2}+\sinh ^{2} \frac{u_{2}}{2}\right) r_{1}-4 r_{2}
\end{array}\right),
$$

where $c$ is the ratio $c=\Omega / \tilde{\Omega}$ we defined in the previous subsection and we introduced

$$
r_{1}=r\left(y_{13}^{-1}\right), \quad r_{2}=r\left(\bar{y}_{21}+\bar{y}_{34}+\bar{y}_{21} y_{13} \bar{y}_{34}\right) .
$$

With a bit of rewriting it follows that

$$
c \frac{\mathcal{K}_{4}}{\Omega} \chi \hat{\pi}^{-1}=\frac{x_{12} x_{13}}{x_{24}^{2}} \frac{1}{4\left((1+U-V)^{2}-4 U\right)}\left(\begin{array}{c}
2 r_{1}+(1+U-V) r_{2} \\
-2(1+U-V) r_{1}-4 U r_{2}
\end{array}\right) .
$$

Thus, we need to show

$$
\left(\begin{array}{l}
r_{1} \hat{\rho}^{-1} \\
r_{2} \hat{\rho}^{-1}
\end{array}\right) M\left(\begin{array}{ll}
T_{1} T_{2}
\end{array}\right)=\frac{x_{24}^{2}}{x_{12} x_{34}}\left(\begin{array}{cc}
4 U & 1+U-V \\
-2(1+U-V) & -2
\end{array}\right),
$$

and this is easily confirmed, supporting our results for tensor structures of 4-dimensional seed blocks. This concludes our comparison of tensor structures for 3- and 4-dimensional seed blocks. Even though the steps were a bit technical, we hope that our discussion helps to better appreciate the similarities of our group theoretic approach with more standard constructions of tensor structures. While the resulting matrix factors can be related by some simple transformations $\chi$, our approach does provide an algorithm for arbitrary spin assignments and it ensures that the associated Casimir equations take the form of eigenvalue equations for some spinning Calogero-Sutherland Hamiltonian. 


\section{Conclusions and outlook}

In this work we employed conformal group theory to explicitly embed the theory of Calogero-Sutherland models and their wave functions into conformal field theory. In the case relevant for four-point functions of spinning fields, Calogero-Sutherland wave functions depend on two variables $u_{i}, i=1,2$, which can be considered as coordinates on a particular 2-dimensional abelian subgroup of the conformal group, as described in eq. (3.1). The precise relation to the usual cross ratios $z$ and $\bar{z}$ had been inferred from the comparison between Casimir equations and eigenvalue equations for Calogero-Sutherland models in [18]. Here we gave a first principle derivation. Let us note that the (exponentials of the) coordinates $u_{i}$ had appeared as radial coordinates in the work of Hogervorst and Rychkov [27] before their relevance for the relation with Calogero-Sutherland models had been appreciated. According to the general discussion in section 2, any function of the two variables $u_{i}$ can be extended to a function of $4 d$ variables $x_{i}$ that satisfies all conformal Ward identities. This extension only depends on the choice of a channel, i.e. an element $\sigma$ of the permutation group $S_{4}$. The map that provides the extension from the cross ratios to the set of insertion points naturally splits into two factors, one that is associated with the subgroup $\mathrm{SO}(1,1)$ of dilations and another associated with the rotation group. The former is a scalar function $\tilde{\Omega}$ that had also been inferred before from the comparison of Casimir and Calogero-Sutherland equations [18] and was now computed independently. The latter function $\Theta$ is matrix valued. More specifically it maps the space of four-point tensor structures to the space of polarizations of the four fields. In analogy to the standard treatments of spinning correlation functions we referred to $\Theta$ as tensor structures. The main new result of this work was to compute $\Theta$ by means of the Cartan decomposition of the conformal group. This was first described in general in section 3 and then carried out explicitly for a few cases in section 4. While our central formula (1.3) resembles similar decompositions in standard treatments of spinning correlators, it is entirely group theoretical in nature and very universal since all Casimir equations in conformal field theory possess a Calogero-Sutherland formulation. By now the latter is known for quite a few cases in which Casimir equations have never been worked out, see also below.

As we stressed in the previous paragraph, the extension of a function $\psi$ of the two variables $u_{i}$ to a function of $4 d$ variables $x_{i}^{\mu}$ depends on the choice of a channel, i.e. a permutation $\sigma \in S_{4}$ of four points in the $d$-dimensional space. Passing (crossing) from one channel $\sigma$ to another $\sigma^{\prime}$ involves the following crossing factors

$$
\Xi_{\sigma^{\prime}}^{\hat{\pi},+}\left(x_{i}\right) \Xi_{\sigma}^{\hat{\pi}}\left(x_{i}\right): W_{P}^{B} \mapsto W_{P}^{B}
$$

where $\Xi^{+}: W_{P} \mapsto W_{P}^{B}$ denotes a left-inverse of $\Xi$. One may think of the product (6.1) as a square matrix of cross ratios. For scalar fields of equal weight $\Delta_{i}=\Delta$ the transition element from the $s$ - to the $t$-channel, i.e. with $\sigma=\sigma_{s}=$ id and $\sigma^{\prime}=\sigma_{t}=(24)$, reads

$$
\Xi_{t}^{\Delta,+}\left(x_{i}\right) \Xi_{s}^{\Delta}\left(x_{i}\right)=\left[\frac{z_{1} z_{2}}{\left(1-z_{1}\right)\left(1-z_{2}\right)}\right]^{\frac{d}{2}-\frac{1}{4}-\Delta}=\left[\frac{U}{V}\right]^{\frac{d}{2}-\frac{1}{4}-\Delta} .
$$

Of course for scalar fields this result is well known, except that we display it here in Calogero-Sutherland gauge rather than the usual conformal one. Since the transition ma- 
trices (6.1) are required to spell out the crossing symmetry constraints for functions of cross ratios it would be interesting to compute these products for spinning blocks. We have not done so in general, but can quote at least one result that applies to the $s=1 / 2$ seed blocks in $d=4$ dimensions. In this case, the crossing factor (6.1) is easy to compute from the explicit expressions (4.28) for $\hat{\pi}$ and eq. (4.26) for $\hat{\rho}$. The result is

$$
\left(\hat{\pi}_{t}\right)^{-1} \hat{\rho}_{t}^{-1} \hat{\rho}_{s} \hat{\pi}_{s}=-i\left(\cosh \frac{u_{1}}{2} \cosh \frac{u_{2}}{2}\right)^{-1 / 2}\left(\begin{array}{cc}
\cosh \frac{u_{1}}{2} & 0 \\
0 & \cosh \frac{u_{2}}{2}
\end{array}\right) .
$$

Here the maps $\hat{\rho}_{t}$ and $\hat{\pi}_{t}$ have been obtained from our expressions for $\hat{\rho}_{s}$ and $\hat{\pi}_{s}$ by exchanging $x_{1}$ and $x_{3}$. The result for the transition matrix is surprisingly simple in this case. It would clearly be of interest to compute such crossing factors for more general spinning blocks.

One of the main motivations for this work was to treat setups in which the tensor structures have not been computed with any other techniques. This applies in particular to the case of superblocks for external operators in long, non-BPS multiplets. For a large class of superconformal symmetries, the Calogero-Sutherland type Casimir equations were recently obtained in [26]. Explicit computations of the corresponding wave functions $\psi$ for $\mathcal{N}=1$ superconformal field theories in $d=4$ dimensions are being worked out in [29]. In order to relate these wave functions to the correlators $G$ it remains to determine the supersymmetric analogues of $\tilde{\Omega}$ and $\Theta$. We will address this problem in future work.

Another important context in which tensor structures are poorly studied concerns defect correlators, i.e. correlation functions that involve one or more non-local operators like line or surface defects etc. Once again, the Calogero-Sutherland models for the relevant wave functions $\psi$ are known from [30], at least in the scalar case. From this work on may also infer the analogue of the factor $\tilde{\Omega}$. Extensions to spinning defect correlators and their blocks have been initiated in [31, 32] and it would be interesting to extend the Calogero-Sutherland approach including our group theoretic construction of the relevant tensor structures $\Theta$ to spinning defect correlators.

\section{Acknowledgments}

We are indebted to Valya Petkova for an interesting conversation that triggered this work. We also thank Giovanni Felder, Madalena Lemos, Pedro Liendo, Slava Rychkov, Aleix Gimenez-Grau and in particular Zhenya Sobko for discussions and comments. This work was supported in parts by the Deutsche Forschungsgemeinschaft (DFG, German Research Foundation) under Germany's Excellence Strategy - EXC 2121 Quantum Universe 390833306. This research has also received funding from the European Research Council (ERC) under the European Union's Horizon 2020 research and innovation program (QUASIFT grant agreement 677368).

\section{A Verification of Ward identities}

The aim of this appendix is to show that the function constructed on the left hand side eq. (2.14) indeed satisfies all the Ward identities a four-point correlation function of spin- 
ning fields has to satisfy. In section 2 we discussed the Ward identities for infinitesimal conformal transformations. Of course, these all integrate to global conformal transformations. Here we shall establish these global Ward identities. Most of our notations were introduced in section 2 already, but we need one more ingredient now concerning the action of global conformal transformations. An element $g \in G$ sends a point $x$ in Euclidean space to $g x$. The differential of $g$ at $x$ will be denoted by $d g_{x}$ - it sends tangent vectors at $x$ to tangent vectors at $g x$. Since the spacetime is just the flat space, we can identify the tangent space at any point with the spacetime itself. After this is done, the statement that $g$ is a conformal transformation means precisely that, at any point $x, d g_{x}$ belongs to the subgroup $K=\mathrm{SO}(1,1) \times \operatorname{Spin}(d)$ that is generated by dilations and rotations of the tangent space. In case $g$ is a translation, $d g_{x}$ is trivial. Since dilations and rotations are linear maps, their differentials are simply the maps themselves (in the obvious sense). For the proof that follows, we will need the differential of the Weyl inversion, which was derived in section 2.1: $d w_{x}=x^{-2} s_{e_{d}} s_{x}$. The global version of the result stated in section 2 reads

Theorem 1. Let $F: G \rightarrow W_{P}$ be a $K \times K$ covariant function satisfying the following covariance law

$$
F\left(k_{l} g k_{r}\right)=\pi_{12}\left(k_{l}\right) \otimes \pi_{34}\left(k_{r}^{-1}\right) F(g),
$$

and define a new function by the right hand side of eq. (2.14), i.e. through the expression

$$
G\left(x_{i}\right)=\frac{1}{x_{12}^{2 \Delta_{2}} x_{34}^{2 \Delta_{4}}}\left(1 \otimes \pi_{2}\left(s_{x_{12}} s_{e_{d}}\right) \otimes 1 \otimes \pi_{4}\left(s_{x_{34}} s_{e_{d}}\right)\right) F\left(e^{I x_{21} \cdot K} e^{x_{13} \cdot P} e^{I x_{34} \cdot K}\right) .
$$

Then for any element $g \in G$ of the conformal group we have

$$
G\left(g x_{i}\right)=\left(\pi_{1}\left(d g_{x_{1}}\right) \otimes \cdots \otimes \pi_{4}\left(d g_{x_{4}}\right)\right) G\left(x_{i}\right) .
$$

Here $g x_{i}$ denotes the image of $x_{i}$ under the action of the global conformal transformation with $g$ and $d g_{x_{i}}$ was introduced in the paragraph preceding this theorem.

Proof. It suffices to show that the claim holds for translations, rotations, dilations and the Weyl inversion $w$, since these generate the whole conformal group. If $g$ is a translation the claim is clear since $d g \equiv 1$ and $G\left(x_{i}\right)$ is manifestly translation invariant. Assume now that $g$ is a dilation $g x=e^{\lambda} x$. Then

$$
\begin{aligned}
G\left(e^{\lambda} x_{i}\right) & =\frac{1}{\left(e^{\lambda} x_{12}\right)^{2 \Delta_{2}}\left(e^{\lambda} x_{34}\right)^{2 \Delta_{4}}} \hat{\rho}\left(e^{\lambda} x_{i}\right) F\left(e^{e^{-\lambda} I x_{21} \cdot K} e^{e^{\lambda} x_{13} \cdot P} e^{e^{-\lambda} I x_{34} \cdot K}\right) \\
& =e^{-2 \lambda\left(\Delta_{2}+\Delta_{4}\right)} \frac{1}{x_{12}^{2 \Delta_{2}} x_{34}^{2 \Delta_{4}}} \hat{\rho}\left(x_{i}\right) F\left(e^{\lambda D} e^{I x_{21} \cdot K} e^{-\lambda D} e^{\lambda D} e^{x_{13} \cdot P} e^{-\lambda D} e^{\lambda D} e^{I x_{34} \cdot K} e^{-\lambda D}\right) \\
& =e^{-2 \lambda\left(\Delta_{2}+\Delta_{4}\right)} e^{-\lambda\left(\Delta_{1}-\Delta_{2}+\Delta_{3}-\Delta_{4}\right)} G\left(x_{i}\right)=\left(\pi_{1}\left(d g_{x_{1}}\right) \otimes \cdots \otimes \pi_{4}\left(d g_{x_{4}}\right)\right) G\left(x_{i}\right) .
\end{aligned}
$$

Here we have used that $\hat{\rho}\left(e^{\lambda} x_{i}\right)=\hat{\rho}\left(x_{i}\right)$. The $\hat{\rho}=\hat{\rho}_{e}$ was defined in eq. (2.16). In the second to last step we have employed the covariance properties (2.13) of the function $F$ and the fact that $\pi_{i}^{\prime}(D)=-\pi_{i}(D)$. Thereby we have established the claim in the case of dilations. 
Next, let $g=r$ be some global rotation of the $d$-dimensional space. In this case one finds that

$G\left(r x_{i}\right)=\frac{1}{x_{12}^{2 \Delta_{2}} x_{34}^{2 \Delta_{4}}}\left(1 \otimes \pi_{2}\left(r s_{x_{12}} r^{-1} s_{e_{d}}\right) \otimes 1 \otimes \pi_{4}\left(r s_{x_{34}} r^{-1} s_{e_{d}}\right)\right) F\left(e^{r I x_{21} \cdot K} e^{r x_{13} \cdot P} e^{r I x_{34} \cdot K}\right)$.

We used the fact that rotations $r \in G$ commute with the conformal inversion $I$. As in the case of dilations, we can manipulate the argument of the function $F$ and insert its covariance properties to obtain

$$
\begin{aligned}
F\left(e^{r I x_{21} \cdot K} e^{r x_{13} \cdot P} e^{r I x_{34} \cdot K}\right) & =F\left(r e^{I x_{21} \cdot K} r^{-1} r e^{x_{13} \cdot P} r^{-1} r e^{I x_{34} \cdot K} r^{-1}\right) \\
& =\left(\pi_{1}(r) \otimes \pi_{2}^{\prime}(r) \otimes \pi_{3}(r) \otimes \pi_{4}^{\prime}(r)\right) F\left(e^{I x_{21} \cdot K} e^{x_{13} \cdot P} e^{I x_{34} \cdot K}\right) .
\end{aligned}
$$

The factor that comes in front of $F$ is combined with the change in the term $\hat{\rho}$

$$
\pi_{2}\left(r s_{x_{12}} r^{-1} s_{e_{d}}\right) \pi_{2}^{\prime}(r)=\pi_{2}\left(r s_{x_{12}} r^{-1} s_{e_{d}} s_{e_{d}} r s_{e_{d}}\right)=\pi_{2}(r) \pi_{2}\left(s_{x_{12}} s_{e_{d}}\right),
$$

and similarly for $\pi_{4}$. Putting the last two calculations together, we conclude that

$$
G\left(r x_{i}\right)=\left(\pi_{1}(r) \otimes \cdots \otimes \pi_{4}(r)\right) G\left(x_{i}\right)=\left(\pi_{1}\left(d g_{x_{1}}\right) \otimes \cdots \otimes \pi_{4}\left(d g_{x_{4}}\right)\right) G\left(x_{i}\right) .
$$

This shows that the function $G$ obeys the same Ward identities for rotations.

It remains to understand special conformal transformations. Instead of addressing them directly, we shall make use of the fact that translations and special conformal transformations are related by the conformal inversion and prove the claim for the Weyl inversion $w$. In evaluating the function $G$ at the points $g x_{i}=w x_{i}$ we shall exploit the following identity in the conformal group

$$
e^{y \cdot P} e^{-I y \cdot K}=w e^{w y \cdot P} s_{e_{d}} s_{y}|y|^{-2},
$$

which is derived in [33], equations (1.27a)-(1.27f). In the following computation, $y$ denotes the point $w x$ that is obtained from $x$ by $w$. Note that this differs from the standard conformal inversion with $I$ by an additional reflection of the sign in the last component. With this in mind we find, using eq. (A.1)

$$
\begin{aligned}
F\left(e^{I y_{21} \cdot K} e^{y_{13} \cdot P} e^{I y_{34} \cdot K}\right) & =F\left(e^{I y_{21} \cdot K} e^{I y_{1} \cdot K} e^{-I y_{1} \cdot K} e^{y_{1} \cdot P} e^{-y_{3} \cdot P} e^{I y_{3} \cdot K} e^{-I y_{3} \cdot K} e^{I y_{34} \cdot K}\right) \\
& =F\left(e^{I y_{21} \cdot K} e^{I y_{1} \cdot K}\left|y_{1}\right|^{2} s_{y_{1}} s_{e_{d}} e^{w y_{1} \cdot P} w w e^{-w y_{3} \cdot P} s_{e_{d}} s_{y_{3}}\left|y_{3}\right|^{-2} e^{-I y_{3} \cdot K} e^{I y_{34} \cdot K}\right) \\
& =F\left(e^{I y_{21} \cdot K} e^{I y_{1} \cdot K}\left|y_{1}\right|^{2} s_{y_{1}} s_{e_{d}} e^{x_{13} \cdot P} s_{e_{d}} s_{y_{3}}\left|y_{3}\right|^{-2} e^{-I y_{3} \cdot K} e^{I y_{34} \cdot K}\right) .
\end{aligned}
$$

Our next step is to commute the dilation and rotation factors all the way to the left and right position so that we can pull them out of the argument with the help of the covariance law (2.13),

$$
\begin{aligned}
& F\left(e^{I y_{21} \cdot K} e^{y_{13} \cdot P} e^{I y_{34} \cdot K}\right)=x_{1}^{2 \Delta_{12}} x_{3}^{2 \Delta_{34}} F\left(e^{\left|y_{1}\right|^{2} I y_{21} \cdot K} e^{y_{1} \cdot K} s_{y_{1}} s_{e_{d}} e^{x_{13} \cdot P} s_{e_{d}} s_{y_{3}} e^{-y_{3} \cdot K} e^{\left|y_{3}\right|^{2} I y_{34} \cdot K}\right) \\
& =x_{1}^{2 \Delta_{12}} x_{3}^{2 \Delta_{34}} F\left(s_{y_{1}} s_{e_{d}} e^{\left(s_{e_{d}} s_{y_{1}}\left|y_{1}\right|^{2} I y_{21}-I x_{1}\right) \cdot K} e^{x_{13} \cdot P} e^{\left(I x_{3}+s_{e_{d}} s_{y_{3}}\left|y_{3}\right|^{2} I y_{34}\right) \cdot K} s_{e_{d}} s_{y_{3}}\right) \\
& =x_{1}^{2 \Delta_{12}} x_{3}^{2 \Delta_{34}}\left(\pi_{12}\left(s_{y_{1}} s_{e_{d}}\right) \otimes \pi_{34}\left(s_{y_{3}} s_{e_{d}}\right)\right) F\left(e^{I x_{21} \cdot K} e^{x_{13} \cdot P} e^{I x_{34} \cdot K}\right) .
\end{aligned}
$$


In the final step we have used that

$$
s_{e_{d}} s_{y_{1}} y_{1}^{2} I y_{21}-I x_{1}=I x_{21},
$$

and a similar equation in order to simplify the argument of the third exponential. Both identities can be verified by a direct calculation. Notice further that

$$
\frac{1}{y_{12}^{2 \Delta_{2}} y_{34}^{2 \Delta_{4}}}=\left(\frac{x_{1}^{2} x_{2}^{2}}{x_{12}^{2}}\right)^{\Delta_{2}}\left(\frac{x_{3}^{2} x_{4}^{2}}{x_{34}^{2}}\right)^{\Delta_{4}} \text {. }
$$

Putting all these pieces together, we have therefore shown that

$$
\begin{aligned}
G\left(y_{i}\right) & =\frac{1}{y_{12}^{2 \Delta_{2}} y_{34}^{2 \Delta_{4}}}\left(1 \otimes \pi_{2}\left(s_{y_{12}} s_{e_{d}}\right) \otimes 1 \otimes \pi_{4}\left(s_{y_{34}} s_{e_{d}}\right)\right) F\left(e^{I y_{21} \cdot K} e^{y_{13} \cdot P} e^{I y_{34} \cdot K}\right) \\
& =\frac{x_{1}^{2 \Delta_{1}} x_{2}^{2 \Delta_{2}} x_{3}^{2 \Delta_{3}} x_{4}^{\Delta_{4}}}{x_{12}^{2 \Delta_{2}} x_{34}^{2 \Delta_{4}}}\left(\pi_{1}\left(s_{y_{1}} s_{e_{d}}\right) \otimes \pi_{2}\left(s_{y_{12}} s_{y_{1}}\right) \otimes \pi_{3}\left(s_{y_{3}} s_{e_{d}}\right) \otimes \pi_{4}\left(s_{y_{34}} s_{y_{3}}\right)\right) F .
\end{aligned}
$$

Whenever we write $F$ without an argument it is understood that is just the element $g\left(x_{i}\right)$. To finish the proof, we make use of the following simple identity for reflections

$$
s_{e_{d}} s_{y_{12}} s_{e_{d}}=s_{I x_{1}-I x_{2}}=s_{x_{1}} s_{x_{12}} s_{x_{2}} .
$$

Along with the property $s_{x} s_{e_{d}}=s_{e_{d}} s_{y}$, we use it to show

$$
s_{y_{12}}=s_{e_{d}} s_{x_{1}} s_{x_{12}} s_{x_{2}} s_{e_{d}}=s_{y_{1}} s_{e_{d}} s_{x_{12}} s_{e_{d}} s_{y_{2}} .
$$

This allows us to evaluate $G\left(y_{i}\right)$ further

$$
\begin{aligned}
G\left(y_{i}\right) & =\frac{x_{1}^{2 \Delta_{1}} x_{2}^{2 \Delta_{2}} x_{3}^{2 \Delta_{3}} x_{4}^{\Delta_{4}}}{x_{12}^{2 \Delta_{2}} x_{34}^{2 \Delta_{4}}}\left(\pi_{1}\left(s_{e_{d}} s_{x_{1}}\right) \otimes \pi_{2}\left(s_{e_{d}} s_{x_{2}} s_{x_{12}} s_{e_{d}}\right) \otimes \pi_{3}\left(s_{e_{d}} s_{x_{3}}\right) \otimes \pi_{4}\left(s_{e_{d}} s_{x_{4}} s_{x_{34}} s_{e_{d}}\right)\right) F \\
& =x_{1}^{2 \Delta_{1}} \cdots x_{4}^{2 \Delta_{4}} \pi_{1}\left(s_{e_{d}} s_{x_{1}}\right) \otimes \cdots \otimes \pi_{4}\left(s_{e_{d}} s_{x_{4}}\right) G\left(x_{i}\right)=\left(\pi_{1}\left(d g_{x_{1}}\right) \otimes \cdots \otimes \pi_{4}\left(d g_{x_{4}}\right)\right) G\left(x_{i}\right) .
\end{aligned}
$$

Once again, we have arrived at the transformation rule under conformal inversions that is obeyed by four-point functions of spinning fields in a conformal field theory, see eq. (2.5). This completes the proof of the theorem.

\section{B Correlators from harmonic analysis}

In section 2 and the previous appendix we have seen how any $K \times K$ covariant function on the conformal group gives a solution to the Ward identities. Now we will show that the converse is also true, i.e. that every solution of the Ward identities arises in this way. The discussion will closely follow that of [20], only being a bit more explicit.

Let $G_{4}:\left(\mathbb{S}^{d}\right)^{4} \rightarrow W_{P}$ be one solution of the Ward identities. It extends to a unique function $F_{4}: G^{4} \rightarrow W_{P}$ with the following properties

$$
F_{4}: G^{4} \rightarrow W_{P}, \quad F_{4}\left(p_{i} g_{i}\right)=\left(\bigotimes_{i=i}^{4} \pi_{i}\left(p_{i}\right)\right) F_{4}\left(g_{i}\right), \quad F\left(e^{x_{i} \cdot P}\right)=G\left(x_{i}\right) .
$$


Here, $p$ denotes an element of the parabolic subgroup $P \subset G$ which is generated by rotations, dilations and special conformal transformations. Representations $\pi_{i}$ of $K$ are extended to those of $P$ by acting trivially with the special conformal transformations. The space of functions with covariance properties of $F_{4}$ is by definition the tensor product of (possibly non-unitary ${ }^{7}$ ) principal series representations $\rho_{i}$ of $G$. Each of the principal series is realised on the space of left-covariant vector-valued functions on the group. For details on the principal series, see [20,21,33]. The four-fold tensor product will be denoted by $\rho=\bigotimes_{i=1}^{4} \rho_{i}$, and its carrier space by $V$.

One can alternatively realise a principal series representation on the space of right covariant functions. If we do this for the second two representations, the element $F_{4}$ is mapped to the function

$$
F_{2, \hat{2}}: G^{4} \rightarrow W_{P}, \quad F_{2, \hat{2}}\left(g_{1}, g_{2}, g_{3}, g_{4}\right)=F_{4}\left(g_{1}, g_{2}, g_{3}^{-1}, g_{4}^{-1}\right) .
$$

By the theorem 9.5 of [33], the tensor product of two principal series representations is isomorphic to a representation induced from the subgroup $K$ of rotations and dilations. The isomorphism takes a function $H_{2}: G^{2} \rightarrow V_{1} \otimes V_{2}$ which belongs to the first space to the function

$$
H_{1}: G \rightarrow V_{1} \otimes V_{2}, \quad H_{1}(g)=H_{2}(g, g w),
$$

where $w$ is the Weyl inversion. Here, it is assumed that representations are realised on spaces of right-covariant functions. For the case of left-covariant functions, the isomorphism takes the form $H_{1}(g)=H_{2}(g, w g)$.

Making use of these isomorphisms, we see that the four-fold tensor product $\rho$ can be realised on the space of functions

$$
F_{1, \hat{1}}: G^{2} \rightarrow W_{P}, \quad F_{1, \hat{1}}\left(k_{l} g_{1}, g_{2} k_{r}\right)=\left(\pi_{12}\left(k_{l}\right) \otimes \pi_{34}\left(k_{r}^{-1}\right)\right) F_{1, \hat{1}}\left(g_{1}, g_{2}\right) .
$$

The relation with between $F_{1, \hat{1}}$ and $F_{2, \hat{2}}$ is given by

$$
F_{1, \hat{1}}\left(g_{1}, g_{2}\right)=F_{2, \hat{2}}\left(g_{1}, w g_{1}, g_{2}, g_{2} w\right) \text {. }
$$

To arrive at the space of $K \times K$ covariant functions we need to trace the conditions on $G_{4}$ implied by the Ward identities through the maps above. For $F_{4}$ they read

$$
F_{4}\left(g_{1} g, g_{2} g, g_{3} g, g_{4} g\right)=F_{4}\left(g_{1}, g_{2}, g_{3}, g_{4}\right) \text {. }
$$

Therefore $F_{1, \hat{1}}$ obeys $F_{1, \hat{1}}\left(g_{1}, g_{2}\right)=F_{1, \hat{1}}\left(g_{1} g, g^{-1} g_{2}\right)$. That is, $F_{1, \hat{1}}$ depends only on the product of its two arguments. Let

$$
F: G \rightarrow W_{P}, \quad F(g)=F_{1, \hat{1}}(g, e),
$$

The map $G_{4} \mapsto F$ establishes an isomorphism of vector spaces $V \rightarrow \Gamma_{\sigma}^{\pi_{i}}$, where $V$ is the space of solutions to the Ward identities. This completes the proof of the claim.

\footnotetext{
${ }^{7}$ Indeed, as is well-known, the representations appearing in physical Euclidean CFT correlators are not unitary from the $\operatorname{Spin}(1, d+1)$ point of view, but are continuations of unitary positive energy representations of $\tilde{\mathrm{SO}}(2, d)$.
} 
We end this appendix with a couple of remarks. The inverse of the map that we just constructed is of course given by (2.14). This follows from the Ward identities. One could also arrive at the answer directly by inverting each of the isomorphisms from above. In this procedure, the only non-trivial input is the inverse of the isomorphism (B.1), which is explicitly written in [33] and is essentially based on the identity (A.1). It is therefore not surprising that this identity played the crucial role in the proof of the Ward identities. We shall not the write the direct derivation here since it adds little to the current discussion, but an enthusiastic reader is invited to verify that it reproduces formula (2.14).

\section{Derivation of eqs. (3.9) and (3.12)}

In this appendix we shall compute the coordinates $u_{1}\left(x_{i}\right)$ and $u_{2}\left(x_{i}\right)$ as well as $\lambda_{l}\left(x_{i}\right)$ and $\lambda_{r}\left(x_{i}\right)$ in the Cartan decomposition of the elements $g\left(x_{i}\right) \in G$ that we defined in eq. (3.8). The results were stated in eqs. (3.9) and (3.12). Since we can work in any faithful representation of $G=\operatorname{Spin}(1, d+1)$, let us take the vector representation by $(d+2) \times(d+2)$ matrices.

Theorem 2. Take four points belonging to Euclidean configuration space on $\mathbb{R}^{d}$, i.e.

$$
\left(x_{a}\right)_{a=1}^{4} \in\left(\mathbb{R}^{d}\right)^{4} \backslash\left\{x_{a} \neq x_{b} \forall x_{a} \neq x_{b}, a, b=1, \ldots, 4\right\} .
$$

Then for $d \geq 2$, the following decomposition holds: ${ }^{8}$

$$
g\left(x_{i}\right):=e^{I x_{21} \cdot K} e^{x_{13} \cdot P} e^{I x_{34} \cdot K}=e^{\lambda^{(l)} D} \cdot r_{l}\left(\psi^{(l)}\right) \cdot e^{\frac{u_{1}}{2}\left(A_{+}-i A_{-}\right)+\frac{u_{2}}{2}\left(A_{+}+i A_{-}\right)} \cdot r_{r}\left(\psi^{(r)}\right) \cdot e^{\lambda^{(r)} D},
$$

where, in particular, ${ }^{9}$

$$
e^{u_{i}}=1-\frac{2}{z_{i}}\left(1+\sqrt{1-z_{i}}\right), \quad i=1,2
$$

and

$$
e^{2 \lambda^{(l)}}=\frac{x_{12}^{2} x_{14}^{2}}{x_{24}^{2}} \frac{1}{\sqrt{\left(1-z_{1}\right)\left(1-z_{2}\right)}}, \quad e^{2 \lambda^{(r)}}=\frac{x_{14}^{2}}{x_{13}^{2} x_{34}^{2}} \frac{1}{\sqrt{\left(1-z_{1}\right)\left(1-z_{2}\right)}} .
$$

Here we switched to usual cross-ratio variables

$$
U=z_{1} z_{2}=\frac{x_{12}^{2} x_{34}^{2}}{x_{13}^{2} x_{24}^{2}}, \quad V=\left(1-z_{1}\right)\left(1-z_{2}\right)=\frac{x_{14}^{2} x_{23}^{2}}{x_{13}^{2} x_{24}^{2}},
$$

so that Euclidean kinematics in the configuration space translates to $z_{1}=z_{2}^{*}, \quad z_{i} \in \mathbb{C}$. This factorization is unique, up to Spin $(d-2)$ redundancy reducing the number of independent angles among $\psi^{(l)}, \psi^{(r)}$ to $d(d-1)-(d-2)(d-3) / 2=\left(d^{2}+3 d-6\right) / 2$.

\footnotetext{
${ }^{8}$ For almost all such $g$, more precisely the singular subvariety is of codimension $d$.

${ }^{9}$ The map (C.2): $\left(u_{1}, u_{2}\right) \rightarrow\left(z_{1}, z_{2}\right)$ is double-covering, since we can choose two different branches of a square root (doing it consistently for both $u_{i}$, the overall number of choices is two).
} 
Proof. Clearly, both sides are elements of $\operatorname{Spin}(1, d+1)$, so we just need to compare the entries of these matrices. One can directly see that the upper left $2 \times 2$ block of the left hand side is of the form

$$
\Gamma=\frac{1}{2}\left(\begin{array}{c}
x_{13}^{2}+\frac{x_{23}^{2}}{x_{12}^{2}}+\frac{x_{14}^{2}}{x_{34}^{2}}+\frac{x_{24}^{2}}{x_{12}^{2} x_{34}^{2}}-x_{13}^{2}+\frac{x_{23}^{2}}{x_{12}^{2}}-\frac{x_{14}^{2}}{x_{34}^{2}}+\frac{x_{24}^{2}}{x_{12}^{2} x_{34}^{2}} \\
x_{13}^{2}-\frac{x_{23}^{2}}{x_{12}^{2}}+\frac{x_{14}^{2}}{x_{34}^{2}}-\frac{x_{24}^{2}}{x_{12}^{2} x_{34}^{2}}-x_{13}^{2}+\frac{x_{23}^{2}}{x_{12}^{2}}+\frac{x_{14}^{2}}{x_{34}^{2}}-\frac{x_{24}^{2}}{x_{12}^{2} x_{34}^{2}}
\end{array}\right),
$$

whereas the corresponding block of the right hand side looks as

$\tilde{\Gamma}=\left(\begin{array}{cc}\cosh u_{12}^{+} \cosh \lambda_{l} \cosh \lambda_{r}+\cosh u_{12}^{-} \sinh \lambda_{l} \sinh \lambda_{r} & \cosh u_{12}^{+} \cosh \lambda_{l} \sinh \lambda_{r}+\cosh u_{12}^{-} \sinh \lambda_{l} \cosh \lambda_{r} \\ \cosh u_{12}^{+} \sinh \lambda_{l} \cosh \lambda_{r}+\cosh u_{12}^{-} \cosh \lambda_{l} \sinh \lambda_{r} & \cosh u_{12}^{+} \sinh \lambda_{l} \sinh \lambda_{r}+\cosh u_{12}^{-} \cosh \lambda_{l} \cosh \lambda_{r}\end{array}\right)$,

with $u_{12}^{ \pm}=\left(u_{1} \pm u_{2}\right) / 2$. Noticing that

$$
U=\frac{4}{\left(\Gamma_{11}-\Gamma_{22}\right)^{2}-\left(\Gamma_{12}-\Gamma_{21}\right)^{2}}, \quad V=\frac{\left(\Gamma_{11}+\Gamma_{22}\right)^{2}-\left(\Gamma_{12}+\Gamma_{21}\right)^{2}}{\left(\Gamma_{11}-\Gamma_{22}\right)^{2}-\left(\Gamma_{12}-\Gamma_{21}\right)^{2}}
$$

and comparing to the left hand side, we obtain eq. (C.2). Noticing that

$$
\begin{aligned}
& 4 \frac{\sqrt{V}}{U} \cosh \left(2 \lambda_{r}\right)=\Gamma_{11}^{2}+\Gamma_{12}^{2}-\Gamma_{21}^{2}-\Gamma_{22}^{2}, \\
& 4 \frac{\sqrt{V}}{U} \cosh \left(2 \lambda_{l}\right)=\Gamma_{11}^{2}-\Gamma_{12}^{2}+\Gamma_{21}^{2}-\Gamma_{22}^{2}
\end{aligned}
$$

and comparing to the left hand side, we obtain eq. (C.3).

Computing the $\left(d^{2}+3 d-6\right) / 2$ Euler angles for rotational matrices $r_{l}$ and $r_{r}$ requires to compare further matrix elements beyond the upper left corner. It is more difficult to obtain closed formulas for these angles. In section 4, however, we compute the six Euler angles for the 3-dimensional case, using the 4-dimensional representation of the conformal group rather than the fundamental one. Extending this type of analysis for higher dimensional conformal groups is possible, but beyond the scope of this work.

\section{Euler angles and quaternions}

To understand the group-theoretic origin of the map (4.15) a bit better, let us first recall that for the 3-dimensional conformal group, the following chain of group isomorphisms holds

$$
\operatorname{Spin}_{\mathbb{R}}(1,4) \simeq\left(\mathcal{C} \ell_{\mathbb{R}}^{0}\right)^{\times}(1,4) / \mathbb{R}^{+} \simeq \mathcal{C} \ell_{\mathbb{R}}^{\times}(1,3) / \mathbb{R}^{+} \simeq \mathbb{H}^{2 \times 2} / \mathbb{R}^{+} \equiv \mathrm{SL}(2, \mathbb{H}) .
$$

Here $\mathcal{C} \ell_{\mathbb{R}}^{0}$ denotes an even part of the corresponding Clifford algebra ${ }^{10}$ over the real numbers and $\left(\mathcal{C} \ell^{0}\right)_{\mathbb{R}}^{\times}$denotes its subgroup of invertible elements. The first isomorphism follows from

\footnotetext{
${ }^{10}$ We use a 'plus sign' convention in definition of the Clifford algebra, i.e. such that the ideal to mod out of tensor algebra $T(V)$ of the underlying vector space $V$ is generated by relation $v \cdot v=q(v) 1$, with $v \in V$ and $q$ quadratic form on $V$.
} 
the definition of the Spin group and is a low-dimensional exception, ${ }^{11}$ while the projection on its right hand side instructs us to take only elements of unit norm. The second comes from a standard isomorphism of Clifford algebras implemented by taking products of a chosen gamma matrix with the rest of those as a basis for the underlying vector space on the right hand side. The third isomorphism, where the $\mathbb{H}^{2 \times 2}$ denotes the group of invertible $2 \times 2$ quaternionic matrices, is implemented via the usual recursive construction of gamma matrices

$$
\mathcal{C} \ell_{\mathbb{R}}(1,3) \simeq \mathcal{C} \ell_{\mathbb{R}}(0,2) \otimes \mathcal{C} \ell_{\mathbb{R}}(1,1) \simeq \mathbb{H} \otimes \mathbb{R}(2) \simeq \mathbb{H}(2),
$$

where all isomorphisms are those of algebras and $\mathbb{R}(n), \mathbb{H}(n)$ denote the corresponding $n \times n$ matrix algebra over reals/quarternions. Indeed, in the realization (4.2) that we chose in section 4 one can directly see eq. (D.1) after multiplying the off-diagonal $2 \times 2$ blocks by $i$.

Let us use the standard matrix presentation for a quaternion, i.e. an element of $\mathbb{H} \simeq$ $\mathcal{C} \ell_{\mathbb{R}}(0,2):^{12}$

$$
X=x_{0}+x_{1} \mathbf{I}+x_{2} \mathbf{J}+x_{3} \mathbf{K}=\left(\begin{array}{cc}
x_{0}+i x_{1} & x_{2}+i x_{3} \\
-x_{2}+i x_{3} & x_{0}-i x_{1}
\end{array}\right),
$$

where the imaginary units $\mathbf{I}, \mathbf{J}, \mathbf{K}$ all square to -1 , anticommute and satisfy $\mathbf{I J}=\mathbf{K}$, $\mathbf{J K}=\mathbf{I}, \mathbf{K I}=\mathbf{J}$. In this language, the rotation matrices $L$ and $L^{\prime}$ (as well as $R$ an $R^{\prime}$ ) are unit quaternions (versors) related by the conjugation with $\mathbf{I}, L^{\prime}=-\mathbf{I} L \mathbf{I} \equiv L^{\mathbf{I}}$. In our chosen conventions this can be also viewed as the involution $t$ of Clifford algebra $\mathcal{C} \ell_{\mathbb{R}}(0,2)$ reverting the signs of odd elements. Since $R$ and $L$ have unit norm, $L^{-1}=\bar{L}$ and $R^{-1}=\bar{R}$, where the bar denotes the conjugation anti-involution of $\mathbb{H}$.

Quaternion multiplication from the left and from the right is clearly an $\mathbb{R}$-linear operation, so it can be implemented by acting with $4 \times 4$ real matrices on vectors of quaternion parameters. Explicitly, these are well-known maps,

$$
\begin{aligned}
& v: \mathbb{H} \rightarrow \mathbb{R}^{4}, \quad X \mapsto\left(x_{0}, x_{1}, x_{2}, x_{3}\right)^{T} \\
& \iota_{L}: \mathbb{H} \rightarrow \mathbb{R}(4), \quad A \mapsto\left(\begin{array}{cccc}
a_{0} & -a_{1} & -a_{2} & -a_{3} \\
a_{1} & a_{0} & -a_{3} & a_{2} \\
a_{2} & a_{3} & a_{0} & -a_{1} \\
a_{3} & -a_{2} & a_{1} & a_{0}
\end{array}\right) \\
& \iota_{R}: \mathbb{H} \rightarrow \mathbb{R}(4), \quad B \mapsto\left(\begin{array}{cccc}
b_{0} & -b_{1} & -b_{2} & -b_{3} \\
b_{1} & b_{0} & b_{3} & -b_{2} \\
b_{2} & -b_{3} & b_{0} & b_{1} \\
b_{3} & b_{2} & -b_{1} & b_{0}
\end{array}\right),
\end{aligned}
$$

\footnotetext{
${ }^{11}$ To render it isomorphism for Euclidean conformal groups of $d>4$ dimensions, an additional condition should be imposed of taking only those elements of the Clifford group whose corresponding inner automorphisms preserve the vector space of gamma matrices. For low-dimensional Spin groups this is automatic, see [34].

${ }^{12}$ This isomorphism is not natural. We use the convention that the underlying vector space of $\mathcal{C} \ell_{\mathbb{R}}(0,2)$ is spanned by basis vectors which are images of $\mathbf{J}$ and $\mathbf{K}$.
} 
where $A, B, X \in \mathbb{H}$. The properties of these maps [35] (lemma 1.23) are in particular such that

- $\iota_{L}\left(A_{1} A_{2}\right)=\iota_{L}\left(A_{1}\right) \iota_{L}\left(A_{2}\right), \iota_{R}\left(B_{1} B_{2}\right)=\iota_{R}\left(B_{2}\right) \iota_{R}\left(B_{1}\right)$

- $\iota_{L}(A) \iota_{R}(B)=\iota_{R}(B) \iota_{L}(A)$

- $v(A X B)=\iota_{L}(A) \iota_{R}(B) v(X)$.

One can understand them more conceptually by embedding both $\mathbb{H}$ and $\mathbb{R}^{4}$ into $\mathcal{C} \ell_{\mathbb{R}}(4,0)$ [36]. Let us introduce the twisted operation $\overline{\iota_{R}}(B)=\iota_{R} \circ \bar{B}$, such that $\overline{\iota_{R}}\left(B_{1} B_{2}\right)=\overline{\iota_{R}}\left(B_{1}\right) \overline{\iota_{R}}\left(B_{2}\right)$. Now we see that the map $v$ turns the algebra of quaternions considered as a bimodule over itself into a left $\mathbb{H} \otimes \mathbb{H}$ module

$$
(A, B) \cdot v(X)=\iota_{L}(A) \overline{\iota_{R}}(B) v(X) .
$$

Since we can do that for any of the four quarternions in $\mathbb{H}^{2 \times 2}$, this takes care of finding a map $\tilde{p}$ that has a property $\tilde{p}\left(r_{l} a r_{r}\right)=L \otimes\left(R^{\prime}\right)^{-1} \tilde{p}\left(g_{a}\right)$ columnwise, see also [37]. Requiring that also $\tilde{p}\left(e^{\lambda_{1} D} r_{l} a r_{r} e^{\lambda_{2} D}\right)=\tilde{p}\left(r_{l} a r_{r}\right) \cdot D_{1}$ chooses an arrangement of the four $4 \times 1$ blocks next to each other and fixes $p$ uniquely, as in the formula (4.15).

Open Access. This article is distributed under the terms of the Creative Commons Attribution License (CC-BY 4.0), which permits any use, distribution and reproduction in any medium, provided the original author(s) and source are credited.

\section{References}

[1] F.A. Dolan and H. Osborn, Conformal four point functions and the operator product expansion, Nucl. Phys. B 599 (2001) 459 [hep-th/0011040] [inSPIRE].

[2] F.A. Dolan and H. Osborn, Conformal partial waves and the operator product expansion, Nucl. Phys. B 678 (2004) 491 [hep-th/0309180] [INSPIRE].

[3] S. Ferrara, A.F. Grillo, G. Parisi and R. Gatto, The shadow operator formalism for conformal algebra. Vacuum expectation values and operator products, Lett. Nuovo Cim. 4 (1972) 115 [INSPIRE].

[4] R. Rattazzi, V.S. Rychkov, E. Tonni and A. Vichi, Bounding scalar operator dimensions in $4 D$ CFT, JHEP 12 (2008) 031 [arXiv:0807.0004] [INSPIRE].

[5] S. El-Showk, M.F. Paulos, D. Poland, S. Rychkov, D. Simmons-Duffin and A. Vichi, Solving the 3D Ising Model with the Conformal Bootstrap, Phys. Rev. D 86 (2012) 025022 [arXiv: 1203.6064] [INSPIRE].

[6] S. El-Showk, M.F. Paulos, D. Poland, S. Rychkov, D. Simmons-Duffin and A. Vichi, Solving the $3 d$ Ising Model with the Conformal Bootstrap II. c-Minimization and Precise Critical Exponents, J. Stat. Phys. 157 (2014) 869 [arXiv:1403.4545] [inSPIRE].

[7] D. Simmons-Duffin, A Semidefinite Program Solver for the Conformal Bootstrap, JHEP 06 (2015) 174 [arXiv: 1502.02033] [INSPIRE].

[8] F. Kos, D. Poland, D. Simmons-Duffin and A. Vichi, Precision Islands in the Ising and $O(N)$ Models, JHEP 08 (2016) 036 [arXiv:1603.04436] [INSPIRE]. 
[9] M.S. Costa, J. Penedones, D. Poland and S. Rychkov, Spinning Conformal Blocks, JHEP 11 (2011) 154 [arXiv: 1109.6321] [INSPIRE].

[10] D. Simmons-Duffin, Projectors, Shadows, and Conformal Blocks, JHEP 04 (2014) 146 [arXiv: 1204.3894] [INSPIRE].

[11] M.S. Costa and T. Hansen, Conformal correlators of mixed-symmetry tensors, JHEP 02 (2015) 151 [arXiv:1411.7351] [INSPIRE].

[12] M.S. Costa, T. Hansen, J. Penedones and E. Trevisani, Projectors and seed conformal blocks for traceless mixed-symmetry tensors, JHEP 07 (2016) 018 [arXiv: 1603.05551] [INSPIRE].

[13] M.S. Costa, T. Hansen, J. Penedones and E. Trevisani, Radial expansion for spinning conformal blocks, JHEP 07 (2016) 057 [arXiv: 1603.05552] [INSPIRE].

[14] D. Karateev, P. Kravchuk and D. Simmons-Duffin, Weight Shifting Operators and Conformal Blocks, JHEP 02 (2018) 081 [arXiv: 1706.07813] [INSPIRE].

[15] J.-F. Fortin, W.-J. Ma, V. Prilepina and W. Skiba, Efficient Rules for All Conformal Blocks, arXiv: 2002.09007 [INSPIRE].

[16] L. Iliesiu, F. Kos, D. Poland, S.S. Pufu, D. Simmons-Duffin and R. Yacoby, Fermion-Scalar Conformal Blocks, JHEP 04 (2016) 074 [arXiv:1511.01497] [INSPIRE].

[17] A. Castedo Echeverri, E. Elkhidir, D. Karateev and M. Serone, Seed Conformal Blocks in 4D CFT, JHEP 02 (2016) 183 [arXiv:1601.05325] [INSPIRE].

[18] M. Isachenkov and V. Schomerus, Superintegrability of d-dimensional Conformal Blocks, Phys. Rev. Lett. 117 (2016) 071602 [arXiv: 1602.01858] [INSPIRE].

[19] G.J. Heckman and E.M. Opdam, Root systems and hypergeometric functions. I, Compos. Math. 64 (1987) 329.

[20] V. Schomerus, E. Sobko and M. Isachenkov, Harmony of Spinning Conformal Blocks, JHEP 03 (2017) 085 [arXiv: 1612.02479] [INSPIRE].

[21] V. Schomerus and E. Sobko, From Spinning Conformal Blocks to Matrix Calogero-Sutherland Models, JHEP 04 (2018) 052 [arXiv:1711.02022] [INSPIRE].

[22] M. Isachenkov, P. Liendo, Y. Linke and V. Schomerus, Calogero-Sutherland Approach to Defect Blocks, JHEP 10 (2018) 204 [arXiv: 1806.09703] [INSPIRE].

[23] M. Isachenkov and V. Schomerus, Integrability of conformal blocks. Part I. Calogero-Sutherland scattering theory, JHEP 07 (2018) 180 [arXiv:1711.06609] [INSPIRE].

[24] M. Isachenkov and V. Schomerus, Integrability of conformal blocks. Part II. Algebraic structure and q-deformation, in preparation.

[25] F.A. Dolan and H. Osborn, Conformal Partial Waves: Further Mathematical Results, arXiv:1108.6194 [INSPIRE].

[26] I. Burić, V. Schomerus and E. Sobko, Superconformal Blocks: General Theory, JHEP 01 (2020) 159 [arXiv: 1904.04852] [INSPIRE].

[27] M. Hogervorst and S. Rychkov, Radial Coordinates for Conformal Blocks, Phys. Rev. D 87 (2013) 106004 [arXiv:1303.1111] [INSPIRE].

[28] G.F. Cuomo, D. Karateev and P. Kravchuk, General Bootstrap Equations in 4D CFTs, JHEP 01 (2018) 130 [arXiv:1705.05401] [INSPIRE]. 
[29] I. Burić, V. Schomerus and E. Sobko, Superconformal Blocks: 4-dimensional $N=1$ theories, in preparation.

[30] P. Liendo, Y. Linke and V. Schomerus, A Lorentzian inversion formula for defect CFT, arXiv:1903.05222 [INSPIRE].

[31] E. Lauria, M. Meineri and E. Trevisani, Spinning operators and defects in conformal field theory, JHEP 08 (2019) 066 [arXiv: 1807.02522] [INSPIRE].

[32] N. Kobayashi and T. Nishioka, Spinning conformal defects, JHEP 09 (2018) 134 [arXiv: 1805.05967] [INSPIRE].

[33] V.K. Dobrev, G. Mack, V.B. Petkova, S.G. Petrova and I.T. Todorov, Harmonic Analysis on the n-Dimensional Lorentz Group and Its Application to Conformal Quantum Field Theory, in Lecture Notes in Physics 63, Springer-Verlag (1977).

[34] H. Lawson and M. Michelsohn, Spin Geometry (PMS-38), Princeton University Press (1989).

[35] K. Gürlebeck and W. Sprössig, Quaternionic and Clifford Calculus for Physicists and Engineers, Wiley (1997).

[36] C. Shao, H. Li and L. Huang, Basis-free Solution to General Linear Quaternionic Equation, arXiv: 1707.00685.

[37] D. Janovska and G. Opfer, Linear equations in quaternionic variables, Mitt. Math. Ges. Hamburg 27 (2008) 223. 